Portland State University

PDXScholar

$12-1-1992$

\title{
Correction of Chromatic Aberration with an Electron Mirror
}

Michael Stewart Mauck

Portland State University

Follow this and additional works at: https://pdxscholar.library.pdx.edu/open_access_etds

Part of the Environmental Sciences Commons, and the Physics Commons Let us know how access to this document benefits you.

\section{Recommended Citation}

Mauck, Michael Stewart, "Correction of Chromatic Aberration with an Electron Mirror" (1992).

Dissertations and Theses. Paper 1171.

https://doi.org/10.15760/etd.1170

This Dissertation is brought to you for free and open access. It has been accepted for inclusion in Dissertations and Theses by an authorized administrator of PDXScholar. Please contact us if we can make this document more accessible: pdxscholar@pdx.edu. 


\title{
CORRECTION OF CHROMATIC ABERRATION
}

\section{WITH AN ELECTRON MIRROR}

\author{
by \\ MICHAEL STEWART MAUCK
}

A dissertation submitted in partial fulfillment of the requirements for the degree of

\section{DOCTOR OF PHILOSOPHY}

in

ENVIRONMENTAL SCIENCES AND RESOURCES:

PHYSICS

Portland State University

1993 


\section{TO THE OFFICE OF GRADUATE STUDIES:}

The members of the Committee approve the dissertation of

Michael Stewart Mauck presented December 1, 1992.

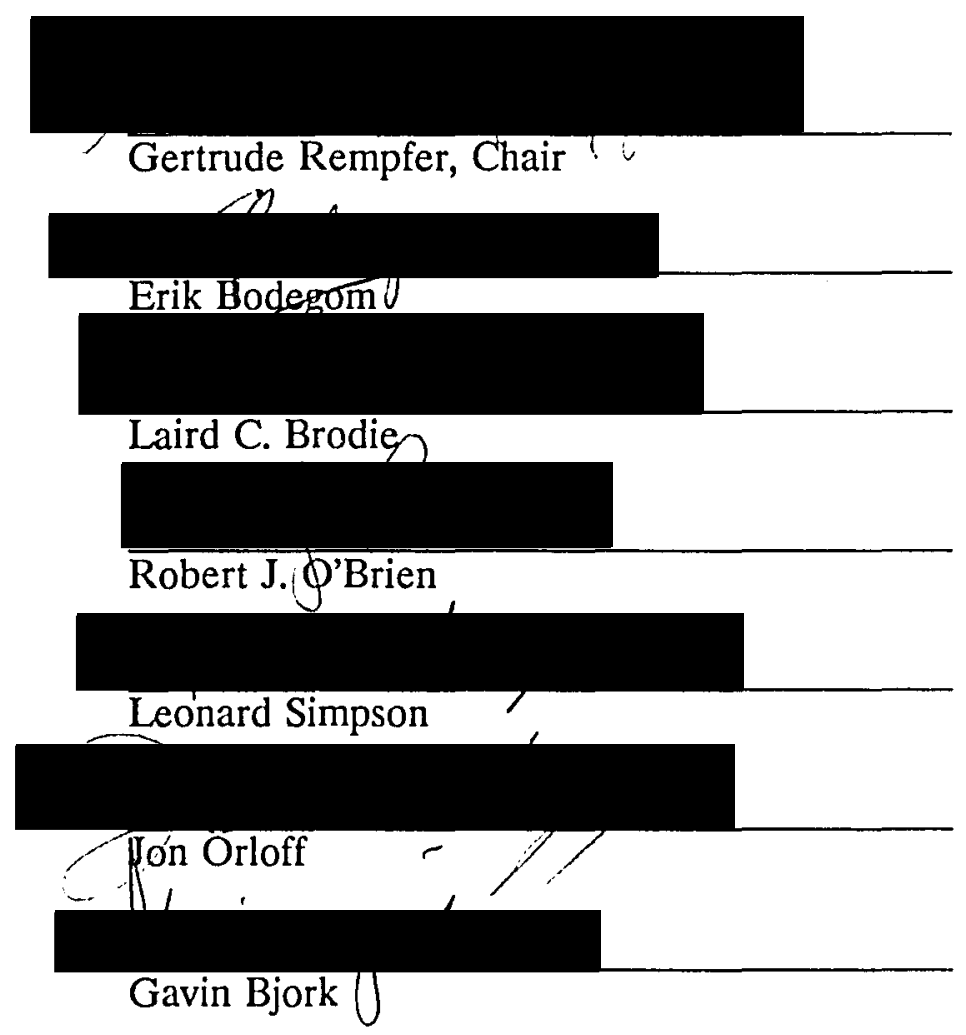

APPROVED:

John/G. Rueter, Director, Environmental Sciences and Resources Program

Roy W. Kogh, Vice Provost for Graduate Studies and Research 
AN ABSTRACT OF THE DISSERTATION OF Michael Stewart Mauck for the Doctor of Philosophy in Environmental Sciences and Resources: Physics presented December 1, 1992.

Title: Correction of Chromatic Aberration with an Electron Mirror.

APPROVED BY THE MEMBERS OF THE DISSERTATION COMMITTEE:

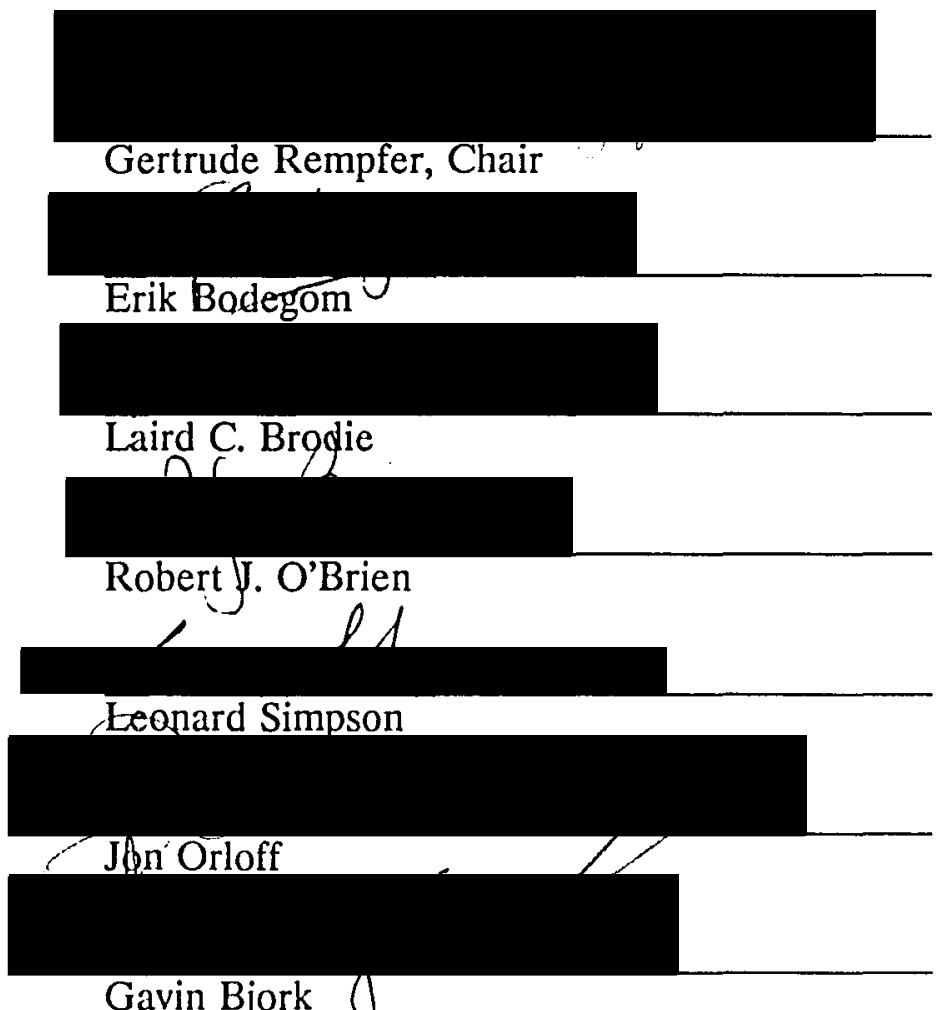

Gavin Bjork O 
The theoretical basis for using electron mirrors as correctors of chromatic aberration is presented and an experimental verification of correction of chromatic aberration is demonstrated. A hyperbolic electrostatic electron mirror operating in its converging range and at unity magnification was used as a corrector. A novel separating system with deflections taking place at image planes was developed to implement the mirror without impairing the resolution. Correction was demonstrated in an electron optical probe system. The chromatic aberration was measured by means of the shadows cast by a fine mesh placed near the final image. The experimental method and equipment are described. The experiment serves as a verification of the theory as well as a successful test of the method of separating the electron beams traveling to and from the mirror. 


\section{ACKNOWLEDGMENTS}

The author is deeply indebted to Dr. Gertrude Rempfer for her untiring interest in her students and her work. Her genius has been a constant source of inspiration for this work as well as a lifetime of research possibilities. Many others contributed to the accomplishment of this project. Dr. O. Hayes Griffith at U. of O. and his capable staff provided the PEM application background as well as administrative and financial assistance. I thank Polly Habliston for the prompt German literature references. Rudy Zupan and the late Garo Arakelyan of the Science Support Shop at PSU were responsible for the precision machining of the many electron optical elements needed in these experiments. Walter Skoczylas and Denis Desloge were of invaluable assistance in preparing the text and figures. The financial assistance of the M. J. Murdock Charitable Trust and gifts from my Mother made this dissertation possible. For the contents of Chapter III, the authors thank Dr. O.H. Griffith for valuable discussions, W.P. Skoczylas and D.M. Desloge for expert engineering and technical support, and David Ryan for photographic services. Financial support from the National Science Foundation (Grant No. DIR8907619) is gratefully acknowledged. 
TABLE OF CONTENTS

PAGE

ACKNOWLEDGMENTS $\ldots \ldots \ldots \ldots \ldots \ldots \ldots \ldots \ldots \ldots$ iii

LIST OF TABLES $\ldots \ldots \ldots \ldots \ldots \ldots \ldots \ldots \ldots \ldots \ldots \ldots \ldots \ldots \ldots \ldots$ vi

LIST OF FIGURES $\ldots \ldots \ldots \ldots \ldots \ldots \ldots \ldots \ldots \ldots \ldots \ldots \ldots \ldots \ldots$ vii

\section{CHAPTER}

I BACKGROUND $\ldots \ldots \ldots \ldots \ldots \ldots \ldots \ldots \ldots \ldots$

Introduction $\ldots \ldots \ldots \ldots \ldots \ldots \ldots \ldots \ldots \ldots \ldots \ldots$

Historical $\ldots \ldots \ldots \ldots \ldots \ldots \ldots \ldots \ldots, 4$

Light Optics

Electron Optics

II THEORETICAL $\ldots \ldots \ldots \ldots \ldots \ldots \ldots \ldots \ldots \ldots \ldots$

Introduction $\ldots \ldots \ldots \ldots \ldots \ldots \ldots \ldots \ldots \ldots$

The Electric Field Equations . . . . . . . . . . . 13

The Hyperbolic Electron Mirror $\ldots \ldots \ldots \ldots \ldots \ldots$

Calculation of the Properties of the

Hyperbolic Electron Mirror for

Symmetric Rays

Electron Trajectories in the Hyperbolic Field

Deflection by the Aperture Lens

The Paraxial Object/Image Distance and the Spherical and Chromatic Aberration

Coefficients of the Mirror 
III CORRECTION OF CHROMATIC ABERRATION WITH AN ELECTRON MIRROR $\ldots \ldots \ldots \ldots \ldots \ldots$

Introduction $\quad \ldots \ldots \ldots \ldots \ldots \ldots \ldots \ldots \ldots \ldots \ldots \ldots \ldots$

The Electron Optical System . . . . . . . . . 32

Method of Determining Aberrations ........ 34

Apparatus .................. 37

Results and Discussion $\ldots \ldots \ldots \ldots \ldots \ldots \ldots$

IV SIGNIFICANCE OF RESULTS ... . . . . . . . . 47

Transmission Electron Microscope $\ldots \ldots \ldots \ldots \ldots 49$

Emission Microscope $\ldots \ldots \ldots \ldots \ldots \ldots \ldots \ldots$

Electron or Ion Probe .............. 52

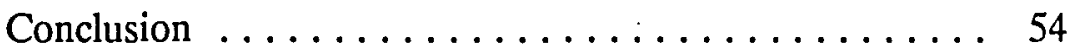

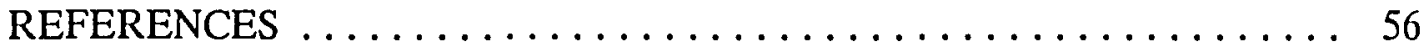

APPENDICES

A THE BEAM-SEPARATING SYSTEM ........... 59

B DETAILS OF THE EXPERIMENT ........... 65 


\section{LIST OF TABLES}

TABLE

PAGE

I Mirror and Lenses $\ldots \ldots \ldots \ldots \ldots \ldots \ldots \ldots 46$

II Projection Lens Properties $\ldots \ldots \ldots \ldots \ldots \ldots \ldots 71$ 


\section{LIST OF FIGURES}

FIGURE

PAGE

1. Construction of elemental volume element

for obtaining divergence of electric

field $\ldots \ldots \ldots \ldots \ldots \ldots \ldots \ldots \ldots \ldots \ldots$

2. Equipotential surfaces for the rotationally

symmetric hyperbolic field $\ldots \ldots \ldots \ldots \ldots 20$

3. Theoretical model of the hyperbolic mirror $\ldots \ldots \ldots .21$

4. Trajectory of the symmetric ray in the hyperbolic mirror field $\ldots \ldots \ldots \ldots \ldots \ldots 24$

5. Theoretical (a) paraxial object/image

distance and (b) aberrations as a

function of the voltage ratio for the

hyperbolic electron mirror in the

symmetric mode $\ldots \ldots \ldots \ldots \ldots \ldots \ldots, 28$

6. The electron optical arrangement used for the

experimental study of aberration correction

by an electron mirror $\ldots \ldots \ldots \ldots \ldots \ldots, 33$ 
7. Diagram illustrating the relation between the magnification of the shadow cast by the mesh and the location of the image formed by the objective lens 35

8. Photograph of the aberration-correction experiment set-up in the electron optical bench $\ldots \ldots \ldots \ldots \ldots \ldots \ldots \ldots$

9. A scale drawing of the hyperbolic mirror used in the experiments $\ldots \ldots \ldots \ldots \ldots \ldots \ldots, 40$

10. A scale drawing of the objective lens used in the aberration-correction experiments $\ldots \ldots \ldots \ldots, 41$

11. Shadow patterns indicating several stages of chromatic aberration correction: (a) undercorrection, (b) correction, (c) overcorrection $\ldots \ldots \ldots \ldots, 42$

12. Shadow pattern obtained under conditions similar to those for Figure 11, but without the correcting system $\ldots \ldots \ldots \ldots \ldots \ldots \ldots, 45$

13. Separation of incident and returning beams by a magnetic field $\ldots \ldots \ldots \ldots \ldots \ldots \ldots \ldots, 61$

14. Schematic of power supplies for experimental study . . . 68 


\section{CHAPTER I}

\section{BACKGROUND}

\section{INTRODUCTION}

The objective of this work is the development of a method of correcting chromatic aberration in an electron optical system by means of a hyperbolic electrostatic electron mirror. The research reported in this dissertation demonstrates the first experimental verification of the theory of aberration correction with mirrors. This achievement provides the basis for extending the resolution limit of electron microscopes and should result in new and important discoveries in all branches of science.

In light optics, chromatic aberration manifests itself as a change in lens power and image position as a function of the wavelength or color used to form the image. We are all familiar with the phenomenon of color dispersion from the occurrences of the rainbow or the colored cusp formed in light passing through a glass of water and falling on the table. In these every day examples of dispersion, light of differing colors is refracted by differing amounts. In the case of lenses, the dispersion of light results in concentric circles of color being formed around each image point, blurring the image and limiting the resolution of the image. 
The invention of the achromat has permitted the correction of this primary defect in light optics.

In electron optics a difference in the velocities of electrons passing through an electron lens causes an analogous behavior. This velocity aberration is also referred to as chromatic aberration in analogy with light optics. Here our every day experience is limited, but aberration of electron beams, for example, sets the upper limit to the brightness in projection television tubes. In electron optics, unlike light optics, there is no rotationally symmetric lens element having the opposite sign of chromatic aberration. However, an electron mirror does have chromatic aberration of opposite sigin from that of electron lenses. By incorporating a mirror in a lens system to compensate the lens aberrations, the entire system can be made free, to first order, from chromatic aberration.

Work on mirrors has been hindered in the past by the need for a practical means of separating the beams headed toward and away from the mirror. The first scheme proposed for incorporating a mirror as a correcting element required the beam to pass through the object twice, compromising the intended benefits. In the work reported here an efficient optical arrangement for incorporating a mirror element in an optical system is demonstrated and confirms the mirror's potential as a correcting element in a variety of electron optical devices.

Demonstration of the correction of chromatic aberration in electron optics provides the basis for improved resolution and larger beam currents in electron and ion optical devices. Correction of chromatic aberration is of particular 
interest in the case of probe-forming instruments, especially focused ion beam instruments, where the energy spread is large [1], and also in emission electron microscopes and related low-energy electron microscopes, where the accelerating field has a large chromatic aberration coefficient $[2,3]$.

Aberration correction would be especially beneficial to the photoelectron microscope (PEM) where direct imaging of the DNA molecule lies near the resolution limit of this instrument [2]. In the photoelectron microscope, photoelectrons are emitted from the specimen by exposure to ultraviolet light. The photoelectrons are accelerated and focussed into a magnified image of the object. The relatively gentle action of the ultraviolet light in the PEM compared to direct electron bombardment of the specimen in the TEM results in less damage to the objects under study. The combination of photoelectric yield and topographic contrast, with the surface specific nature of image formation in the PEM is advantageous for biological imaging. Improving the resolution from the present limit of about $100 \AA$ to $20 \AA$ or less would enable direct imaging of an important new range of biological structures in the PEM.

Ion beam spectrometers and probe type instruments could also incorporate this chromatic correction to increase resolution and sensitivity to minute environmental contaminants. Increasing need to extend the limits of detection in analytical instruments will stimulate the design of new improved instruments incorporating the electron mirror. Furthermore, the microelectronics industry is constantly moving toward producing integrated circuits with smaller features. 
Presently the lithography industry is reaching the resolution limit of light optics and is investigating the possibility of utilizing $x$-rays to reach smaller sizes. Increased resolution in electron and ion optics provides an alternative to the use of x-rays for production and analysis of smaller geometries. Another entirely new branch of micro-machining fabrication called "nanotechnology" has developed around lithography and micro-machining utilizing ion probes. Both resolution and throughput of ion beam probes are limited by electron lens aberrations. Correction of chromatic and/or spherical errors with the electron mirror will revolutionize this field. These are a few of the anticipated benefits from the correction of the primary defects in electron optics. The demonstration of the correction of chromatic aberration described in this work is a significant step in realizing these objectives.

\section{HISTORICAL}

\section{Light Optics}

Correction of chromatic aberration in light optics was accomplished over two hundred years ago. Spurred on by the mistaken belief that the eye is achromatic Chester Moor Hall is credited [4] with constructing the first achromatic telescope in about 1733 . Later, John Dolland patented a practical achromat in 1758 [5]. The achromat corrects both chromatic and spherical aberration. The achromat enabled larger telescope lenses to be made, spawning the modern age of astronomy. It is difficult to imagine our world today without 
microscopes, cameras, camcorders, copiers, microfilm, and the host of manufacturing processes that depend upon the achromat.

When aberrations have been corrected, the ultimate resolution limit depends upon the diffraction limit due to the wave nature of light. The diffraction limit $r_{d}$ is given by

$$
\mathrm{r}_{\mathrm{d}}=0.61 \lambda /(\mathrm{n} \sin \alpha)
$$

where $\lambda$ is the wave length of light, $\mathrm{n}$ is the index of refraction around the specimen, and $\alpha$ is the maximum angle of light accepted by the optics. The resolution limit is about $3000 \AA$ for visible light.

\section{Electron Optics}

The ultimate limit to resolution in electron optics is also set by the wave nature of electrons to

$$
r_{d}=0.61 \lambda / \sin \alpha
$$

where the wavelength in Angstroms is given by $\lambda=\sqrt{(150 / V)}$ and $V$ is the electron accelerating voltage in volts. The wavelength is $0.05 \AA$ for electrons accelerated through $60 \mathrm{kV}$. The angle $\alpha$ accepted by the lens system for a $60 \mathrm{kV}$ beam is usually quite small, $\propto 0.01 \mathrm{rad}$. resulting in a resolution limit of $3 \AA$. The small angle is a consequence of a compromise between the diffraction error, which increases as the angle decreases, and the lens aberration error which increases as the angle increases. In this case we see that the $3 \AA$ resolution limit is nowhere near the $0.05 \AA$ wavelength of the electrons. The case for ions with 
their shorter wavelengths corresponding to their greater mass is even more disparate. The potential gains in resolution in electron and ion beams by the correction of aberrations is large and many have undertaken to accomplish this goal.

The earliest work published on electron mirrors was done by Henneberg and Recknagel [6], Recknagel [7], and Hottenroth [8]. The discovery that mirrors had the opposite sign of chromatic aberration from lenses was apparently Henneberg's as he applied for a patent in May 1935 in Germany and was subsequently granted a U.S. patent [9] in 1939. The book by Zworkin et al. [10], published in 1947, also demonstrated conclusively that even the flat electrostatic mirror was overcorrected for both spherical and chromatic aberrations.

In Scherzer's celebrated paper [11] of 1936, he proved that the chromatic and spherical errors of conventional electron lenses were always of the same sign. Efforts to reduce aberrations had, by this time, resulted in the growing concern that the enormous resolution potential of electron microscopy could be only partially realized.

In another paper [12] Scherzer delineated the possible ways of overcoming aberrations in electron lenses. The list contains the various conditions under which corrections are theoretically possible in a rotationally symmetric electron lens system. Each of these methods involves some kind of intrusion into the space in which the electron beam would normally travel in an uncorrected system. In principle spherical aberration can be corrected by the use of multipole electric 
or magnetic fields. The multipole fields are used to remove the rotational symmetry of an electron optical system. It is then necessary to restore the rotational symmetry after correction of spherical aberration $[13,14]$. Both spherical and chromatic aberrations can, in principle, be corrected by combining electric and magnetic poles in the corrector [15]. Another approach is to construct a diverging correcting element. This can be done by utilizing a conducting foil window which is nearly transparent to electrons on one or more electrodes of an electrostatic lens. Here the diverging component is combined with an undercorrected converging lens to form a spherically corrected converging doublet $[16,17]$ which can be partially corrected for chromatic aberration. In principle the field in a lens can also be modified by a space charge injected along the axis of the lens. Time-varying focusing fields and pulsed electron beams can also, in principle, be used to produce lenses corrected for aberrations. Recent reviews of such approaches to the correction of chromatic and spherical aberrations are found in Refs. [18] and [19]. The mirror is the only rotationally symmetrical geometry in Scherzer's list which doesn't require the beam to traverse some physical obstacle or the electric or magnetic field to vary in time. These restrictions are important in preserving the potential of the optical system to produce resolution of atomic dimensions.

The history of the mirror was known to Ramberg, and the publication of the list of possibilities for overcoming aberrations articulated by Scherzer may be what led Ramberg to investigate the possibility of utilizing a mirror to improve 
the resolution of the electron microscope. In 1948 Ramberg [20] proved that the mirror field he studied analytically would have overcorrected chromatic aberration, that is, higher energy electrons would focus closer to the mirror rather than farther away as in the usual case with lenses. In his scheme, the specimen was situated between the objective lens and the mirror. The illuminating beam would have to pass through the specimen on the way to the mirror and again on the way back to the objective. The mirror was used in the symmetrical mode with the magnification near unity. In this case the aberration of the mirror would have to be numerically equal to that of the objective, and the mirror and objective would have to have external focal points. The impracticality of having the beam pass through the specimen a second time as well as the restrictive optical constraints caused Ramberg to conclude that "the correction of electron microscope objectives by electron mirrors, employed in the manner described, meets serious practical difficulties." After Ramberg's statement of the impracticability of using a converging mirror to correct the aberrations of lens systems was published, further work using mirrors for this purpose was not seen for two decades.

However, mirrors were interesting for reasons other than for aberration correction. For example, the development of a commercial energy analyzing electron microscope by Zeiss utilized a mirror as part of the energy filter. An electron mirror utilizing a plane mirror electrode was incorporated into a 
magnetic prism-mirror-prism geometry by Castaing and Henry [21] in 1962 as an element of the high resolution energy filter in this design.

In 1973 Henkelman and Ottensmeyer [22] were investigating a concave mirror geometry for a use similar to the Castaing filter, and made this remark in closing: "The demonstration by Ramberg (1948) that the dimensions of a correcting mirror must be unobtainably small does not apply for images which have been further magnified as in this case. Whether such a correction can be realized practically has not yet been investigated." Although they did not have aberration correction in view in their work, the use of a mirror in their geometry with the image of the specimen at other than unity magnification eliminated one of the objections to the practical realization of the mirror for aberration correction. Mirrors were considered in similar applications by Lichte and Mollenstedt [23] in 1979. It was, in fact, the mirror's potential of large chromatic aberration that was found of interest as a dispersing element in the energy analyzer studied by van der Merwe [24] in 1981.

In my Master's thesis [25] in 1969 both the theoretical and experimental properties of the hyperbolic electrostatic electron mirror were studied. The only previous mention of the hyperbolic field model in the literature is in the limiting case of an analytical model by Lafferty [26] in 1947. In this case only the first order properties were investigated. The hyperbolic field is a particularly instructive system to study since the equations for the radial and axial motion are separable and have analytic solutions. By taking into account the terminating 
aperture lens effect, the theoretical model closely mimics the real mirror and much insight into the behavior of the mirror properties can be understood by studying the theory. This work confirmed the expectation that the converging hyperbolic field possessed aberrations suitable for correction of electron lens systems. No separation scheme was required in my experimental investigation. The mirror was used at a magnification different from unity, and the object and image were at different locations so that the incident and returning beams traveled along different paths. A hole cut in the photographic film used to record the reflected beam permitted the incident beam to reach the mirror while the returning beam was intercepted by the film surrounding the hole. The properties of the mirror could be studied in this way but this scheme would be impractical for a microscope.

The need to separate the incident beam from the reflected beam without impairment of resolution by aberrations of the deflection system remained as an obstacle until the present work. Work toward realizing a practical separation scheme began in the allied field of mirror microscopy. Here, the specimen itself is the mirror, and is the object to be imaged. The incident beam is separated from the reflected beam by a magnetic turning field. The earliest use the magnetic separator is found in the paper by Hottonroth [8] where the mirror is first investigated experimentally. Distortion and aberrations of the turning field used in this way preclude it from use in high resolution aberration correction. Subsequent searching for the lowest aberration magnetic turning fields exhausted 
the potential of bringing these aberrations to a sufficiently low magnitude to use at high resolution. The situation with the separation system was not unlike the quest for the lens with the highest resolution for electron microscopy. No theoretical limit was known for the minimum aberration possible and so the search went on for the best geometry until the possibilities were exhausted.

In order to utilize the aberration correction properties of a mirror, a practical method of separating the incident and reflected beams must be employed. One method of separation that had not been tried is to separate the beams in an image plane. For this case, the object and image must both be superimposed in a separating magnetic turning field. This stipulation requires the mirror to operate in its symmetrical mode with equal object and image distances. The experimental investigation of the mirror's properties in the symmetric mode, in turn, requires the use of the same beam separation scheme where the deflections take place at image planes.

In the present study the equations governing the motion of electrons in the hyperbolic electric field in its symmetric mode are reviewed in Chapter II. Here the theoretical properties are presented utilizing an analytic model for the hyperbolic electron mirror previously published by Dr. Rempfer and myself [27] and Rempfer [28]. In this study a practical high resolution beam separation scheme is developed and used to demonstrate correction of chromatic aberration in a system of lenses by the mirror in Chapter III. The experimental properties of the mirror are deduced from the results of the aberration correction of the system 
and compared with the results of the theoretical study of the mirror properties. Satisfactory correlation between the mirror's theoretical and experimental properties are found. In Chapter IV the significance of the research is discussed and applications where the correction of chromatic aberration would be expected to be beneficial are suggested. The techniques developed to accomplish the beam separation and correction are presented in detail in Appendix A. The separation system developed here has utility beyond aberration correction and has become the subject of further development of the low energy reflection electron microscope [29] and will be useful in other electron and ion instruments. The details of the experimental procedure and equipment are described in Appendix B. Further evidence for the practicality of the lens-mirror achromat found in this study is discussed. 


\section{CHAPTER II}

\section{THEORETICAL}

\section{INTRODUCTION}

To begin I will review the theoretical background leading to the electron optical properties of the hyperbolic electron mirror. This review will include: the equations necessary to describe the motion of electrons in electric fields; the steps leading to the equations for the potential distribution in rotationally symmetric electric fields satisfying Laplace's equation, of which the hyperbolic field is a special case; the solution of the electron trajectories in the hyperbolic field and the electron optical properties of the hyperbolic electron mirror.

\section{THE ELECTRIC FIELD EQUATIONS}

Here we begin from first principles and deduce the equations governing the electrostatic electric field and the motion of electrons in such a field. The essential physical law to begin with is Coulomb's force law

$$
\mathbf{F}=\mathrm{k} \mathrm{q} \mathrm{q} / \mathrm{r}^{2}
$$

where $\mathbf{F}$ is the force between two "point" charges $\mathrm{q}$ and $\mathrm{q}$ ' a distance $\mathrm{r}$ apart.

The electric field $\mathbf{E}$ produced by a charge $\mathrm{q}$ is defined to be the limit of the 
ratio of the force $\mathbf{F}$ on a test charge q' (introduced at the point of interest) to the quantity of charge on the test charge as the magnitude of the test charge approaches zero.

$$
\mathbf{E}=\lim _{q^{\prime} \rightarrow 0}\left(\mathbf{F} / q^{\prime}\right)
$$

In this formalism a charge is said to produce an electric field extending to infinity in all directions in proportion to the magnitude of the charge, inversely proportional to the square of the distance from a point charge and dependent upon the electric properties of the intervening space. The introduction of the concept of the field is of great use in that vector algebra and vector calculus can be brought to bear on the mathematical solutions of practical problems.

A field can be represented by lines of force where the direction of the field is represented by the direction of the lines, and the strength of the field by the number of lines per unit area at right angles to the direction of the field. Because of the inverse square law these lines of force are continuous in charge-free space. The same property holds in free space for the field lines due to a distribution of charge. For a given volume in empty space the number of lines entering is equal to the number leaving the volume by Gauss's law. This can be expressed by $\operatorname{div} \mathbf{E}=0$.

The divergence of a vector is the net excess of lines per unit volume leaving an infinitesimal region. The calculation of the divergence of the electric field is illustrated for Cartesian coordinates in Figure 1. The net flux through the two faces normal to the $\mathrm{x}$-axis is 
$-\left(E_{x} d y d z\right)_{x}+\left(E_{x} d y d z\right)_{x+d x}=\partial\left(E_{x} d y d z\right) / \partial x d x=\partial\left(E_{x} / \partial x\right) d x d y d z$

since $d y$ and $d z$ are independent of $x$. In the same way the flux through the other two pairs of faces can be found, and the total net flux per unit volume is

$$
\left(\partial \mathrm{E}_{\mathrm{x}} / \partial \mathrm{x}+\partial \mathrm{E}_{\mathrm{y}} / \partial \mathrm{y}+\partial \mathrm{E}_{\mathrm{z}} / \partial \mathrm{z}\right)=\operatorname{div} \mathbf{E}
$$

When there are no electric field sources in the volume, $\operatorname{div} \mathbf{E}=0$.

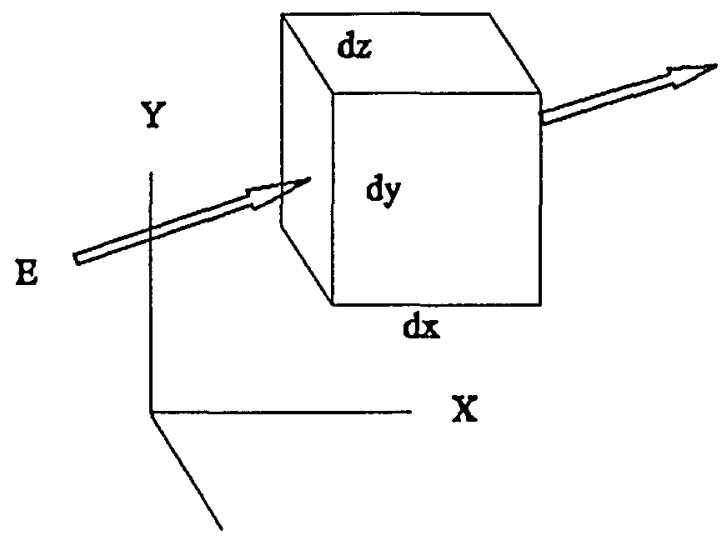

$\mathbf{z}$

Figure 1. Construction of elemental volume element for obtaining divergence of electric field.

If an infinitesimal test charge is moved in an electric field $\mathbf{E}$ a distance $\mathrm{dI}$, the work $\mathrm{dW}$ done against the field by moving the charge is

$$
\mathrm{dW}=-\mathbf{E} \cdot \mathrm{dl} \text {. }
$$

A scalar potential field $\mathrm{V}$ can be defined as the ratio of the work done moving the test charge (from a reference position where $V=0$ to the location where the voltage equals $\mathrm{V}$ ) to the magnitude of the charge. Since the electric force and the electric field obey the law of vector addition, the scalar potential fields add algebraically. The potential field is frequently more convenient to deal with since 
it is a scalar and more easily calculated. The electric field is related to the scalar potential $\mathrm{V}$ by

$$
\mathbf{E}=-\operatorname{grad} \mathrm{V}
$$

The gradient of a scalar is the change in the value of the scalar per unit distance normal to a surface of constant value (i. e., the limit as the distance goes to zero). The gradient of $V$ is the rate of change of potential with distance in the direction normal to the surface at potential V. In Cartesian coordinates

$$
\operatorname{grad} V=\mathbf{i} \partial \mathrm{V} / \partial \mathrm{x}+\mathbf{j} \partial \mathrm{V} / \partial \mathrm{y}+\mathbf{k} \partial \mathrm{V} / \partial z \quad \text { (often written } \nabla \mathrm{V} \text { ) }
$$

That grad $\mathrm{V}$ is normal to the surface = constant can be seen by moving a point a differential amount on an equipotential surface, so that

$$
d V=(\partial V / \partial x) d x+(\partial V / \partial y) d y+(\partial V / \partial z) d z=0
$$

Since the vector $\mathbf{i} d x+\mathbf{j} d y+\mathbf{k d z}$ can take any orientation on the surface, the vector $\mathbf{i} \partial V / \partial x+\mathbf{j} \partial V / \partial y+\mathbf{k} \partial V / \partial z$ must be normal to the surface because the cosine of the angle between the two vectors is zero. Since $\mathbf{E}=-\operatorname{grad} \mathrm{V}$,

$$
\operatorname{div} \mathbf{E}=\operatorname{div}(\operatorname{grad} V)=0 \quad\left(\text { often written } \nabla^{2} V=0\right) .
$$

This is Laplace's equation which tells how the potential function, and hence the electric field, varies in free space for given boundary conditions. In Cartesian coordinates the Laplacian becomes

$$
\nabla^{2} \mathrm{~V}=\partial^{2} \mathrm{~V} / \partial \mathrm{x}^{2}+\partial^{2} \mathrm{~V} / \partial \mathrm{y}^{2}+\partial^{2} \mathrm{~V} / \partial \mathrm{z}^{2}=0
$$

In other coordinate systems the distances corresponding to increments of the coordinates are not necessarily equal to the change in coordinates. Since the hyperbolic mirror field is rotationally symmetric about the optical axis it is 
convenient to express the Laplacian in terms of the cylindrical coordinates $\mathrm{r}$ and z. To do this both div and grad need to be expressed in the cylindrical coordinate system. Expressing the distance increments as $d s_{1}=h_{1} d q_{1}$, etc., $\operatorname{grad} V$ is

$$
\begin{aligned}
& \mathrm{e}_{1} \partial \mathrm{V} / \partial \mathrm{s}_{1}+\mathrm{e}_{2} \partial \mathrm{V} / \partial \mathrm{s}_{2}+\mathrm{e}_{3} \partial \mathrm{V} / \partial \mathrm{s}_{3}= \\
& \mathrm{e}_{1}\left(1 / \mathrm{h}_{1}\right) \partial \mathrm{V} / \partial \mathrm{q}_{1}+\mathrm{e}_{2}\left(1 / \mathrm{h}_{2}\right) \partial \mathrm{V} / \partial \mathrm{q}_{2}+\mathrm{e}_{3}\left(1 / \mathrm{h}_{3}\right) \partial \mathrm{V} / \partial \mathrm{q}_{3}=-\mathbf{E},
\end{aligned}
$$

where the e-vectors are unit vectors in the direction of the increasing coordinates. Similarly, the divergence of $\mathbf{E}$ is

$$
\begin{aligned}
& {\left[\partial / \partial \mathrm{s}_{1}\left(\mathrm{E}_{1} \mathrm{ds}_{2} \mathrm{ds}_{3}\right) \mathrm{ds}_{1}+\partial / \partial \mathrm{s}_{2}\left(\mathrm{E}_{2} \mathrm{ds}_{3} \mathrm{ds}_{1}\right) \mathrm{ds}_{2}\right.} \\
& \left.+\partial / \partial \mathrm{s}_{3}\left(\mathrm{E}_{3} \mathrm{ds}_{1} \mathrm{ds}_{2}\right) \mathrm{ds}_{3}\right] /\left(\mathrm{ds}_{1} \mathrm{ds}_{2} \mathrm{ds}_{3}\right)= \\
& -\left\{\partial / \partial \mathrm{q}_{1}\left[\left(\mathrm{~h}_{2} \mathrm{~h}_{3} / \mathrm{h}_{1}\right) \partial \mathrm{V} / \partial \mathrm{q}_{1}\right]+\partial / \partial \mathrm{q}_{2}\left[\left(\mathrm{~h}_{3} \mathrm{~h}_{1} / \mathrm{h}_{2}\right) \partial \mathrm{V} / \partial \mathrm{q}_{2}\right]\right. \\
& \left.+\partial / \partial \mathrm{q}_{3}\left[\left(\mathrm{~h}_{1} \mathrm{~h}_{2} / \mathrm{h}_{3}\right) \partial \mathrm{V} / \partial \mathrm{q}_{3}\right]\right\} /\left(\mathrm{h}_{1} \mathrm{~h}_{2} \mathrm{~h}_{3}\right) .
\end{aligned}
$$

For cylindrical coordinates $\mathrm{r}, \phi, \mathrm{z}$

$$
\mathrm{ds}_{1}=\mathrm{dr}, \mathrm{ds}_{2}=\mathrm{rd} \phi, \text { and } \mathrm{ds}_{3}=\mathrm{d} z,
$$

and Laplace's equation is

$$
\partial^{2} V / \partial r^{2}+(1 / r) \partial V / \partial r+\left(1 / r^{2}\right) \partial^{2} V / \partial \phi^{2}+\partial^{2} V / \partial z^{2}=0
$$

Since the electric field has rotational symmetry the term involving $\phi$ drops out, leaving

$$
\partial^{2} \mathrm{~V} / \partial \mathrm{r}^{2}+(1 / \mathrm{r}) \partial \mathrm{V} / \partial \mathrm{r}+\partial^{2} \mathrm{~V} / \partial \mathrm{z}^{2}=0
$$

If we assume for $V(r, z)$ a Taylor expansion in powers of $r$ from the axis outward

$$
\begin{gathered}
V(r, z)=V(0, z)+r(\partial V / \partial r)_{0}+\left(r^{2} / 2 !\right)\left(\partial^{2} V / \partial r^{2}\right)_{0} \\
+\left(r^{3} / 3 !\right)\left(\partial^{3} V / \partial r^{3}\right)_{0}+\ldots
\end{gathered}
$$


The requirement that this expression have axial symmetry leads to the following conditions: the odd derivatives with respect to $r$ are zero since $V(r)$ must equal $\mathrm{V}(-\mathrm{r})$; the even derivatives of $\mathrm{V}$ with respect to $\mathrm{r}$ are related to the derivatives with respect to $z$ on the axis, thus

$$
\begin{aligned}
& \left(\partial^{2} \mathrm{~V} / \partial \mathrm{r}^{2}\right)_{0}=-1 / 2 \mathrm{~V}(0, \mathrm{z})^{\prime \prime} \\
& \left(\partial^{\mathrm{n}+2} \mathrm{~V} / \partial \mathrm{r}^{\mathrm{n}+2}\right)_{0}=-(\mathrm{n}+1) /(\mathrm{n}+2)\left(\partial^{\mathrm{n}} \mathrm{V} / \partial \mathrm{r}^{\mathrm{n}}\right)_{0},
\end{aligned}
$$

where the primes refer to derivatives with respect to $z$. When these conditions are used to eliminate the partial derivatives with respect to $r$, the power series expression for $\mathrm{V}$ above becomes

$$
\begin{gathered}
\mathrm{V}(\mathrm{r}, \mathrm{z})=\mathrm{V}(0, \mathrm{z})-\left(\mathrm{r}^{2} / 2^{2}\right) \mathrm{V}(0, \mathrm{z}) "+\left(\mathrm{r}^{4} / 2^{2} 4^{2}\right) \mathrm{V}(0, \mathrm{z})^{\prime \prime \prime} \\
+\left(\mathrm{r}^{6} / 2^{2} 4^{2} 6^{2}\right) \mathrm{V}(0, \mathrm{z})^{(6)}+\ldots
\end{gathered}
$$

and the electric field is

$$
-\mathbf{E}=\nabla V=e_{r} \partial V / \partial r+e_{\phi} \partial V / \partial \Phi+e_{z} \partial V / \partial z .
$$

The radial component of the electric field is

$$
E_{r}=-\partial V / \partial r=1 / 2 V^{\prime \prime}(0, z) r+\text { higher order terms. }
$$

From this equation we see that to first order the radial electric field $E_{r}$ is proportional to the distance $\mathrm{r}$ off axis.

The radial electric field exerts a radial force $F=-\mathrm{E}_{\mathrm{r}}$ on the electron, and from Newton's 2nd law

$$
F_{r}=m \ddot{r}=-e E_{r}=-1 / 2 e V^{\prime \prime}(0, z) r+\ldots
$$

The deflections of the electron trajectories are therefore also proportional to first order to the height off axis. Thus, every rotationally symmetric electric field is a 
focusing field for electrons, unless $V^{\prime \prime}(0, z)=0$, in which case $V$ is either constant, or has a uniform gradient.

\section{THE HYPERBOLIC ELECTRON MIRROR}

The hyperbolic electron mirror is based on the rotationally symmetric hyperbolic potential field as developed in the previous section. From (14) the potential field having cylindrical symmetry and satisfying Laplace's equation can be expressed to first order as

$$
V(r, z)=V(0, z)-V^{\prime \prime}(0, z) r^{2} / 2^{2}+\text { higher order terms }
$$

where again, $\mathrm{r}$ and $\mathrm{z}$ are radial and axial cylindrical coordinates. When the third and higher order derivatives are zero in the field described by (17), and when $V^{\prime \prime}(0, z)=$ a constant $\neq 0$, we are left with the hyperbolic potential field. The integration of $V^{\prime \prime}(0, z)$ with respect to $z$ yields $V(0, z)=C_{1} z^{2} / 2+C_{2} z+C_{3}$. The origin can be chosen so that the equation for the hyperbolic field becomes

$$
V(r, z)-V_{o}=C_{1} z^{2} / 2-C_{1} r^{2} / 2^{2}=k\left(z^{2}-r^{2} / 2\right)
$$

where the potential at the origin is $V_{0}$.

The hyperbolic field yields equipotential surfaces which are hyperboloids of revolution around the $z$ axis. The equipotential surfaces for $V=V_{o}$ are the asymptotic cones of the hyperbolic field, given by $z^{2}-r^{2} / 2=0$, or $z= \pm r / \sqrt{2}$. The vertex of the cones is the origin of coordinates, with the cone axes along the $z$ axis, and the half-angle of the cones given by $\arctan \sqrt{2}$. When $z^{2}-r^{2} / 2>0$ the potential surfaces are two hyperboloidal surfaces intersecting the axis at right 
angles on opposite sides of the origin. When $\mathrm{z}^{2}-\mathrm{r}^{2} / 2<0$ the hyperboloidal surfaces have annular shapes surrounding the axis. The intersections of the equipotential surfaces of the hyperbolic field in a meridional plane containing the axis are shown in Figure 2.

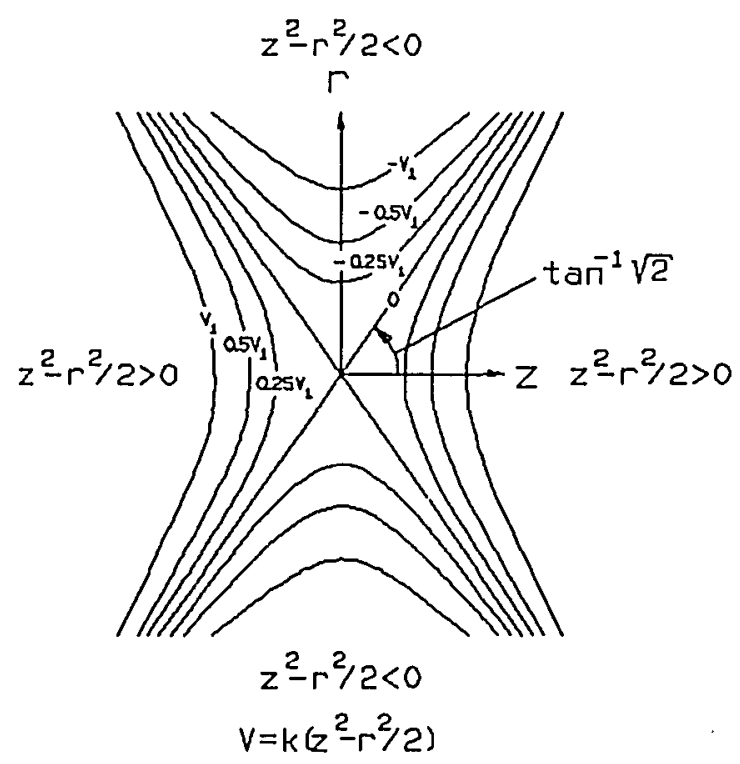

Figure 2. Equipotential surfaces for the rotationally symmetric hyperbolic field. The equipotentials are surfaces of revolution about the $z$ axis. The curves shown are intersections of the surfaces with a plane containing the axis.

In the present study, the shapes of the electrodes in the electron mirror are modeled to approximate the equipotential surfaces of the hyperbolic field. The mirror electrode at a negative potential $V_{M}$ is an asymptotic cone of the hyperbolic field. The anode electrode at a positive potential $V_{A}$ is a hyperboloid of revolution except for a small opening centered on the axis. The electrons enter and exit through this opening. These surfaces are shown in Figure 3. The mirror electrode is more negative than the cathode, so that electrons entering through 
the opening in the anode are turned back before reaching the mirror electrode. The dashed curve in Figure 3 represents an equipotential surface at the same potential $V_{C}$ as the cathode. The field between the mirror electrode and the anode is a hyperbolic field except for the effect of the opening in the anode. The aperture in the anode acts as a thin diverging lens (aperture lens) on the electrons. The mirror field is treated as a hyperbolic field terminated by the aperture lens in the calculations that follow. In the hyperbolic model the electron

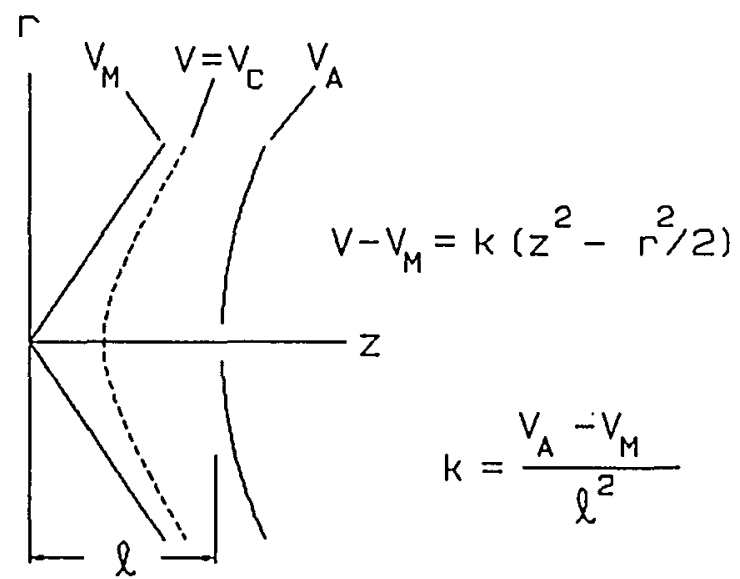

Figure 3. Theoretical model of the hyperbolic mirror. The mirror electrode at potential $\mathrm{V}_{\mathrm{M}}$ is an equipotential asymptotic cone of the hyperbolic field.

trajectories can be calculated analytically. The results can be compared with measurements obtained experimentally for a mirror having essentially the same potential field. Verifying the theoretical model experimentally provides a test of the theory and the physical model of the mirror and will produce confidence in using the theory to correct lens systems. 
Calculation of the Properties of the Hyperbolic Electron Mirror for Symmetric Rays

In the theoretical study, the mirror field is considered to be composed of a hyperbolic field terminated by the aperture lens. Between the anode and mirror electrode the electron trajectories are calculated from the equation of motion of the electrons in the hyperbolic field. As the electrons enter and leave the mirror field they pass through the opening in the anode and are deflected by the aperture lens. Rays beginning at points on the equipotential surface at cathode potential are chosen and the intersection (real or virtual) of the electron rays with the axis, referred to field-free space outside the mirror, are calculated along with the angle of intersection as a function of the initial height of the ray. The electrons leave the cathode potential surface in the mirror field with zero initial velocity and trace out the path of the symmetric ray. Rays are calculated for a number of initial heights for a series of values of the ratio of the accelerating voltage to the mirror voltage. Each voltage ratio corresponds to electrons traveling to and from a different potential surface in the mirror field. Using these calculations, as well as an expression for the paraxial object/image distance $z_{\mathrm{o}}$, the spherical and chromatic aberration coefficients $C_{s}$ and $C_{c}$ are obtained.

\section{Electron Trajectories in the Hyperbolic Field}

In the hyperbolic field of the mirror the potential $\mathrm{V}$ is given by

$$
\mathrm{V}-\mathrm{V}_{\mathrm{M}}=\mathrm{k}\left(\mathrm{z}^{2}-\mathrm{r}^{2} / 2\right)
$$

where the potential $V_{M}$ replaces $V_{o}$ of equation (18), and again, $r$ and $z$ are the 
radial and axial cylindrical coordinates. The coordinate origin is at the vertex of the mirror electrode. The coefficient $\mathrm{k}$ is equal to $\left(\mathrm{V}_{\mathrm{A}}-\mathrm{V}_{\mathrm{M}}\right) / \ell^{2}$ when $\ell$ is the axial length of the mirror field. The equations of motion for the radial and axial components of the electron motion in the hyperbolic field for electron trajectories lying in meridional planes are:

$$
\begin{aligned}
\ddot{r} & =-(\mathrm{ek} / \mathrm{m}) \mathrm{r} \\
\ddot{z} & =2(\mathrm{ek} / \mathrm{m}) \mathrm{z},
\end{aligned}
$$

where e and $\mathrm{m}$ are the charge and mass of the electron, and the second derivatives with respeci to time are marked with two dots.

For the symmetric ray, the electron penetrates the hyperbolic field until it reaches the point $\left(r_{C}, z_{C}\right)$ on the potential surface $V_{C}$, as shown in Figure 4. Here the velocity components $t$ and $z$ are both zero, and if $t$ is chosen to be zero at this point the solutions for the electron coordinates and velocity components as a function of time are:

$$
\begin{aligned}
& r=r_{C} \cos (\omega t) \\
& z=z_{C} \cosh (\sqrt{2} \omega t) \\
& t=-\omega r_{C} \sin (\omega t) \\
& z=\sqrt{2} \omega z_{C} \sinh (\sqrt{2} \omega t),
\end{aligned}
$$

where $\omega=V(e k / m)$. On the cathode potential surface, the coordinates $r_{C}$ and $z_{C}$ satisfy the hyperbolic relation $\mathrm{z}_{\mathrm{C}}{ }^{2}-\mathrm{r}_{\mathrm{C}}{ }^{2} / 2=\mathrm{z}_{\mathrm{Co}_{0}}{ }^{2}$. Here $\mathrm{z}_{\mathrm{Co}_{0}}$ is the axial coordinate of the vertex of the potential surface $V_{C}$, and is given by

$$
\mathrm{z}_{\mathrm{Co}}{ }^{2} / \ell^{2}=\left(\mathrm{V}_{\mathrm{C}}-\mathrm{V}_{\mathrm{M}}\right) /\left(\mathrm{V}_{\mathrm{A}}-\mathrm{V}_{\mathrm{M}}\right)=1-v,
$$


where $v$ is the ratio of accelerating voltage to mirror voltage $\left(V_{A}-V_{C}\right) /\left(V_{A}-V_{M}\right)$.

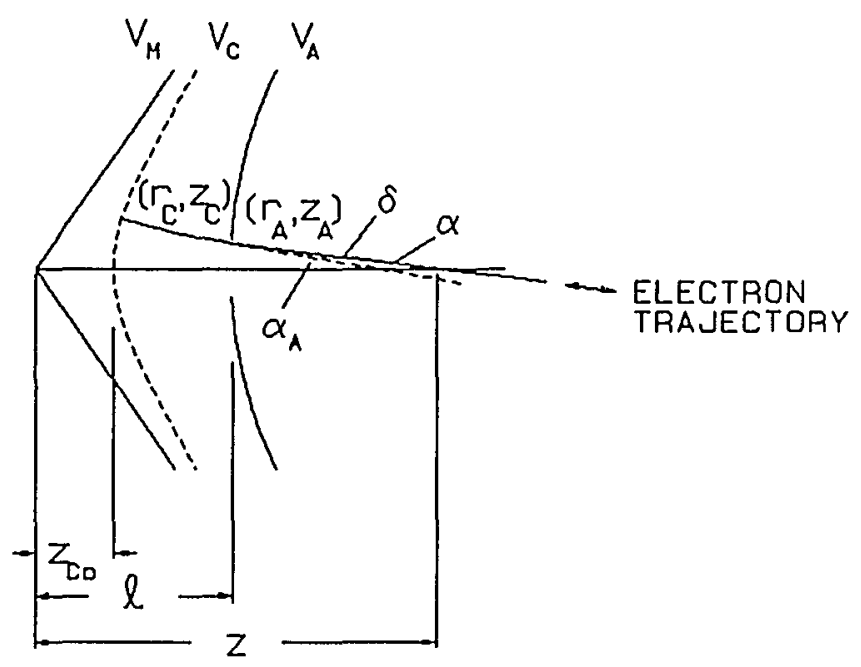

Figure 4. Trajectory of the symmetric ray in the hyperbolic mirror field.

Because of the symmetry of the trajectory described by (22) and (23) we will consider only the returning portion of the trajectory. Beginning at $\left(\mathrm{r}_{\mathrm{C}}, \mathrm{z}_{\mathrm{C}}\right)$ on the surface having potential equal to the cathode potential $V_{c}$, the returning electron reaches the anode at time $t_{A}$ given by

$$
\begin{aligned}
& r_{A}=r_{C} \cos \theta \\
& z_{A}=z_{C} \cosh (\sqrt{2} \theta),
\end{aligned}
$$

where $\theta=\omega \mathrm{t}_{\mathrm{A}}$. This portion of the path of the electron terminates at the point $\left(r_{A}, z_{A}\right)$ on the hyperboloid at anode potential. The coordinates are related by the condition $\mathrm{z}_{\mathrm{A}}^{2}-\mathrm{r}_{\mathrm{A}}^{2} / 2=\ell^{2}$, where $\ell$ is the length of the mirror field along the $\mathrm{z}$ axis. A first approximation for $\theta$ can be obtained by setting $z_{A}=\ell$ in equation (28). With this value of $\theta$ a first approximation for $r_{A}$ is obtained. Using this value in equation (27) yields a new value for $\theta$ which in turn leads to improved 
values of $z_{A}, \theta$, and $r_{A}$. For the narrow beams used in electron optics, one iteration of this sequence is usually enough to provide sufficient accuracy.

When the electron reaches the anode, its velocity components are

$$
\begin{aligned}
& \mathrm{r}_{\mathrm{A}}=-\omega \mathrm{r}_{\mathrm{C}} \sin \theta=-\omega \mathrm{r}_{\mathrm{A}} \tan \theta \\
& \mathrm{z}_{\mathrm{A}}=\sqrt{2} \omega \mathrm{z}_{\mathrm{C}} \sinh (\sqrt{2} \theta)=\sqrt{2} \omega \mathrm{z}_{\mathrm{A}} \tanh (\sqrt{2} \theta) .
\end{aligned}
$$

The slope of the electron trajectory as it reaches the anode is

$$
\mathrm{r}_{\mathrm{A}} / \mathrm{z}_{\mathrm{A}}=\left(-\mathrm{r}_{\mathrm{A}} \tan \theta\right) /\left(\sqrt{2} \mathrm{z}_{\mathrm{A}} \tanh (\sqrt{2} \theta)\right)=-\tan \alpha_{\mathrm{A}} .
$$

Here $\alpha_{\mathrm{A}}$ is the angle which the tangent to the trajectory makes with the axis prior to deflection by the aperture lens (see Figure 4).

\section{Deflection by the Aperture Lens}

The focal length for a circular aperture in an electrode can be calculated by means of the aperture lens formula developed by Davisson-Calbick (D-C) [30]. The D-C formula gives the focal length as

$$
\mathrm{f}_{\mathrm{A}}=4 \mathrm{~V}_{\mathrm{B}} /\left[(\partial \mathrm{V} / \partial \mathrm{z})_{2}-(\partial \mathrm{V} / \partial \mathrm{z})_{1}\right]
$$

where $V_{B}$ is the beam voltage at the plane of the electrode, and the denominator is the difference between the potential gradients at the two faces of the electrode if no aperture were present in the electrode. The D-C formula is good in the weak lens approximation, where the variation in potential across the aperture is small compared with the beam voltage of the electrons. Since the anode in the mirror field is a curved hyperbolic surface, the aperture appears to act as a thin meniscus lens on the surface of the anode. At the anode $V_{B}=V_{A}-V_{C},(\partial V / \partial z)_{2}$ $=0$, and $(\partial \mathrm{V} / \partial z)_{1}=2 \mathrm{kz}_{\mathrm{A}}=2\left(\mathrm{~V}_{\mathrm{A}}-\mathrm{V}_{\mathrm{M}}\right) \mathrm{z}_{\mathrm{A}} / \ell^{2}$, from which 


$$
\mathrm{f}_{\mathrm{A}}=-2\left[\left(\mathrm{~V}_{\mathrm{A}}-\mathrm{V}_{\mathrm{C}}\right) /\left(\mathrm{V}_{\mathrm{A}}-\mathrm{V}_{\mathrm{M}}\right)\right]\left(\ell^{2} / \mathrm{z}_{\mathrm{A}}\right)=-2 v \ell / \eta,
$$

where $\eta=z_{A} / \ell=V\left(1+r_{A}^{2} / 2 \ell^{2}\right)$.

The trajectory of an electron passing through the aperture lens is deflected by an angle

$$
\delta=\arctan \left(\mathrm{r}_{\mathrm{A}} / \mathrm{f}_{\mathrm{A}}\right)=\arctan \left(-\mathrm{r}_{\mathrm{A}} \eta / 2 \nu \ell\right) \text {. }
$$

The deflected trajectory makes an angle

$$
\begin{aligned}
\alpha & =\alpha_{\mathrm{A}}+\delta \\
& =\arctan \left(\mathrm{r}_{\mathrm{A}} \tan \theta / \sqrt{2} \mathrm{z}_{\mathrm{A}} \tanh (\sqrt{2} \theta)\right)-\arctan \left(\mathrm{r}_{\mathrm{A}} \eta / 2 v \ell\right)
\end{aligned}
$$

with the axis in field-free space outside the mirror field. The ray or its virtual extension intersects the axis at $\mathrm{z}$ according to

$$
z-z_{A}=r_{A} / \tan \alpha .
$$

The Paraxial Object/Image Distance and the Spherical and Chromatic Aberration Coefficients of the Mirror

For paraxial rays (36) can be simplified to yield an expression for the paraxial object or image distance $z_{0}$

$$
z_{0} / \ell-1=q_{0}=2 v /\left(\sqrt{2} v \tan \theta_{0}-1\right) \text {. }
$$

The chromatic aberration coefficient is calculated from the variation of $z_{0}$ with electron energy. For the symmetric ray case, equal amounts of chromatic aberration $\Delta_{c} z_{o}$ occur on the object and image side of the mirror. Since the magnification $m$ is equal to unity, the total chromatic aberration, referred to either object or image space, is $\left(\Delta_{c} z_{o}\right)_{\text {total }}=2 \Delta_{c} z_{o}$. The chromatic aberration coefficient $\mathrm{C}_{\mathrm{c}}$ is defined by 


$$
\left(\Delta_{\mathrm{c}} \mathrm{z}_{\mathrm{o}}\right)_{\text {total }}=\mathrm{C}_{\mathrm{c}} \Delta v / v \text {. }
$$

Differentiation of the expression for $z_{0}$ with respect to $v$ in (38) yields the following expression for $\mathrm{C}_{\boldsymbol{c}}$ :

$$
\mathrm{C}_{\mathrm{c}} / \ell=-[v /(1-v)]\left[1+\mathrm{q}_{\mathrm{o}}+(3 / 4) \mathrm{q}_{\mathrm{o}}{ }^{2} / \mathrm{v}^{2}\right]
$$

with $\mathrm{q}_{\mathrm{o}}$ as in (38) and for $\mathrm{m}=1$.

The spherical aberration coefficient $C_{s}$ is obtained from the variation of the object or image distance $z$ with the angle $\alpha$ of the rays. The spherical aberration for symmetric rays is also equally divided between the object and the image, with $\Delta_{\mathrm{s}} \mathrm{z}=\mathrm{z}-\mathrm{z}_{\mathrm{o}}$ on each side. The total aberration $\left(\Delta_{\mathrm{s}} \mathrm{z}\right)_{\text {total }}$ is $2 \Delta_{\mathrm{s}} \mathrm{z}$ and the spherical aberration coefficient $C_{s}$ is defined by

$$
\left(\Delta_{\mathrm{s}} \mathrm{z}\right)_{\text {total }}=-\mathrm{C}_{\mathrm{s}} \alpha^{2} .
$$

The convention of regarding $C_{s}$ as positive for the usual case for lenses where spherical aberration is undercorrected, i.e. for $z-z_{0}<0$ requires the minus sign to appear in (40).

The electron mirror properties calculated from the equations developed here are presented graphically in Figures $5 \mathrm{a}$ and $5 \mathrm{~b}$, as a function of the voltage ratio $v$. The mirror properties are shown only for the first working region, where the incident and returning electrons cross the axis once.

The normalized paraxial object/image distance $z_{0} / \ell$ is plotted in Figure 5a. The distance $z_{0}$ is seen to increase continuously as $v$ decreases over the range of $\checkmark$ plotted. For $v$ closer to 1.0 than shown, the electrons cross the axis more than once. For $v$ near 0.6 , the mirror becomes diverging. The transition from the 

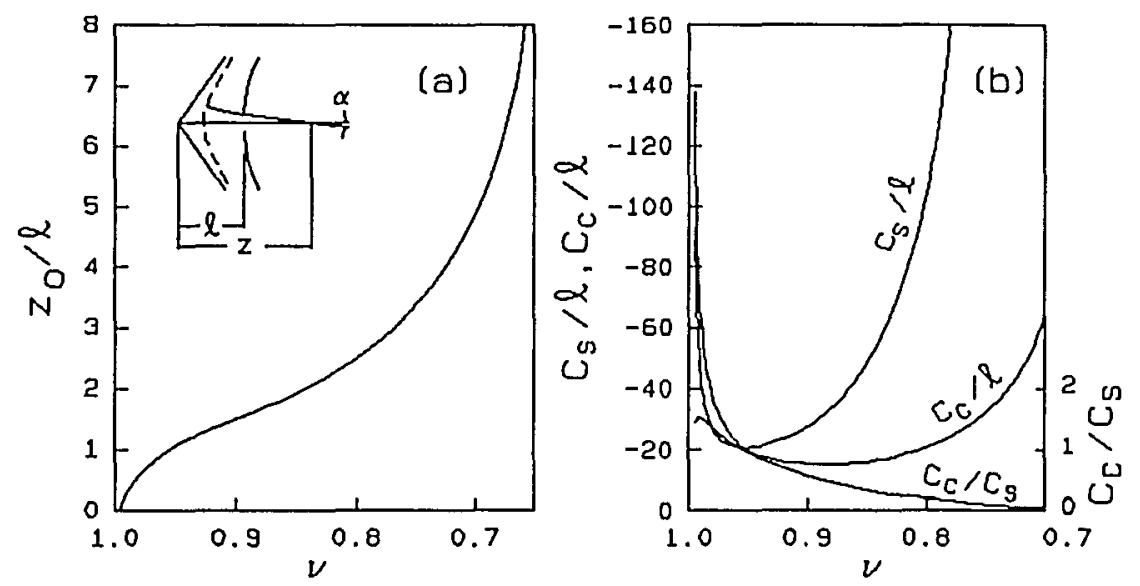

Figure 5. Theoretical (a) paraxial object/image distance and (b) aberrations as a function of the voltage ratio for the hyperbolic electron mirror in the symmetric mode.

converging mode to the diverging mode results from two effects. As $v$ decreases, the converging action of the hyperbolic field becomes weaker since the electrons do not penetrate as far into the field as do electrons with larger values of $v$. At the same time, the diverging action of the aperture lens becomes stronger according to equation 10 because the beam voltage in the numerator is being reduced while the mirror voltage, and hence the field gradient at the aperture in the denominator, remains constant.

The dimensionless normalized coefficients of chromatic and spherical aberration are plotted as $\mathrm{C}_{\mathrm{c}} / \ell$ and $\mathrm{C}_{\mathrm{s}} / \ell$ in Figure $5 \mathrm{~b}$. The ratio of the coefficients $C_{c} / C_{s}$ is also plotted. Both of the aberration coefficients are negative, denoting overcorrection. The magnitudes of both aberrations are large in the regions where $v$ approaches 1.0 and 0.6 , near the ends of the range plotted. In between, there is a broad minimum, but both of the coefficients remain negative 
while their ratio continues to change monotonically.

The reason that the hyperbolic mirror has overcorrected aberration properties can be understood qualitatively as follows. The hyperbolic field produces converging action which depends both on the radial force on the electron, which is proportional to the distance from the axis, and on the time which the electron spends in the field. For chromatic aberration, the lower energy electrons penetrate less far into the field than do higher energy electrons. Lower energy electrons spend less time in the field and are consequently less strongly converged by the field. The action of the aperture lens on the other hand, is more strongly diverging for lower energy electrons. These two effects combine causing the focal distance to be longer for low energy electrons than for high energy electrons. For spherical aberration, electrons entering the mirror offaxis do not penetrate as far into the field. Consequently they spend less time in the hyperbolic field than do paraxial electrons. Thus the converging action is weaker for them, which makes the focal distance longer for off-axis rays than for paraxial rays.

We can now understand why the aberration coefficients increase as $v$ approaches 1.0. The time electrons spend in the mirror field depends on the electron energy and the angle $\alpha$ which the trajectory makes with the axis. For an electron traveling along the axis, the time which it spends in the field approaches $\infty$ as $v$ approaches 1.0. A less energetic electron or an off-axis electron of the same energy spends a finite time in the field. The result is that both chromatic 
and spherical aberrations increase dramatically for the voltage ratio approaching 1. The aberration coefficients also become large as $v$ approaches 0.6 . Here $z_{0}$ is large, as shown in Figure 5a, and $\alpha$ is small; consequently small aberrations in the trajectories produce relatively large longitudinal aberrations. In addition, for the spherical aberration coefficient, a given longitudinal aberration $\Delta z$ produces a larger increase in the aberration coefficient when $\alpha$ is small since $C_{s}=-\Delta z / \alpha^{2}$. In matching the over-corrected aberrations of the mirror to the undercorrected aberrations of a lens or lens system, there are three mirror parameters at our disposal. The voltage ratio $v$ of the mirror, the scale of the mirror, and the magnification of the image to which the mirror correction is applied can all be chosen to effect the match. The ratio of the aberration coefficients, as well as their magnitudes, varies as a function of the voltage ratio $v$, as shown in Figure 5b. The mirror scale, voltage ratio, and magnification must be adjusted in a coordinated way in order to achieve simultaneous correction of chromatic and spherical aberration. Other parameters, associated with the electron optical system to be corrected, include the design and relative scale of the lenses, and their voltage ratios which in turn determine the magnitude of chromatic and spherical error to be corrected. 


\section{CHAPTER III}

\section{CORRECTION OF CHROMATIC ABERRATION WITH AN ELECTRON MIRROR ${ }^{1}$}

\section{INTRODUCTION}

Electron mirrors have chromatic and spherical aberration coefficients of opposite sign from those of electron lenses and, in principle, can be used to compensate the spherical and chromatic aberrations of electron lens systems $[7,20,22,24]$. A method of implementing a hyperbolic electron mirror as an aberration corrector in electron microscopes has been proposed recently [28]. Correction of chromatic aberration is of particular interest in the case of emission electron microscopes and related low-energy electron microscopes, where the accelerating field has a large chromatic aberration coefficient $[2,3]$, and also in probe-forming instruments, especially focused ion beam instruments, where the energy spread is large [1]. In the present paper we report the experimental verification of the correction of chromatic aberration by means of a hyperbolic electron mirror.

1 The contents of this chapter are reproduced from Ref. 31 with permission of the co-author. Minor editing, including renumbering figures and references, was required to harmonize with the text of the dissertation. 


\section{THE ELECTRON OPTICAL SYSTEM}

The electron optical arrangement described in ref. [28] is used in the experimental study. The conditions leading to aberration correction are investigated with the optical system operating in the probe mode, shown schematically in Figure 6. The object for the optical system is the exit pupil (or crossover) of the electron gun. The final image formed by the optical system is a demagnified image of the crossover. Aberration correction is provided by the hyperbolic mirror at the left of the diagram. The electron beam enters the mirror from the right, and is reflected at a curved equipotential surface of the mirror field. The incident and reflected beams are centered on the mirror axis. The mirror is operated in its converging range, and in the symmetric mode, i.e., with object and image distances equal.

In order to independently control the electron beams headed toward and away from the mirror it is necessary to use a beam separator in conjunction with the mirror. The beam separator used in our experiments and described in ref. [28] consists of three magnetic deflectors, two pairs of relay lenses, and an interface lens. The magnetic deflector on the mirror axis performs the function of splitting the incident and reflected beams apart; the other deflectors bend the incident and reflected beams so that their axes are parallel with the mirror axis. A key feature of the beam separator is that deflections take place at image planes, which largely avoids the detrimental effects of deflection aberrations. The 


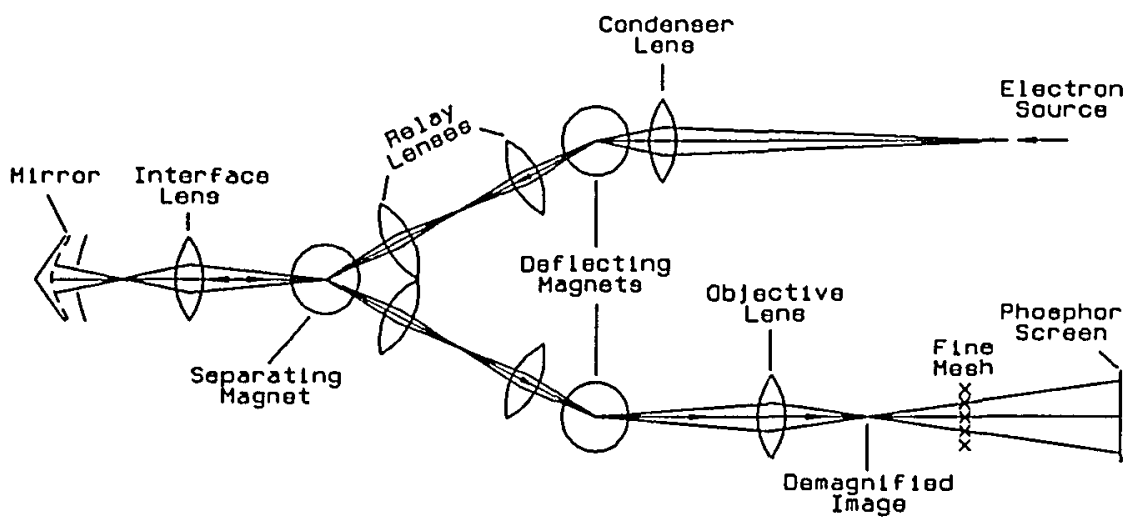

Figure 6. The electron optical arrangement used for the experimental study of aberration correction by an electron mirror. The electron source (upper right) serves as the object for the system. Aberrations in the demagnified image of the source (lower right) are investigated by means of the shadows cast by a fine mesh closely following (or preceding) the image. Aberration correction is provided by the hyperbolic mirror at the left of the diagram. Separation of the beams heading toward and away from the mirror is accomplished by a beam-separator consisting of three magnetic deflectors (at image planes), two pairs of relay lenses, and an interface lens.

relay lenses are used to transfer the image between deflectors. Several other features further reducesensitivity to deflection aberrations: the images at the deflection planes are magnified; the image is transferred between deflectors in parallel orientation; the two deflections in each branch are in opposite senses with respect the image; and the deflection angle is fairly small (about $\left.16^{\circ}\right)$. The interface lens enables the use of magnified images at the deflection planes and serves to balance aberrations by acting as a magnification interface between the objective lens and the mirror. The interface lens is operated in zoom fashion with 
the mirror to keep the images centered in the deflectors during adjustment of the mirror voltage.

\section{METHOD OF DETERMINING ABERRATIONS}

The method of investigating the aberrations in the final image of Figure 6 is based on the grating shadow method of ref. [32]. In the present experiments a fine mesh rather than a bar grating is used to enable us to detect the presence of astigmatism. The mesh is placed closely following (or preceding) the image, and casts a highiy-magnified shadow on a phosphor screen. The shadow is used to determine the image aberrations. The geometrical relation between the shadow magnification $M^{\prime}$ and the image distance $z^{\prime}$ is illustrated in Figure 7. With the mesh located downstream from the image, as in the diagram, and at a distance $d$ from the phosphor screen, the distance $\mathrm{c}$ from the image to the mesh is given by $(c+d) / c=E^{\prime} / e^{\prime}=M^{\prime}$, where $e^{\prime}$ is the height of the ray at the mesh, and $E^{\prime}$ is the height of the shadow. Solving for $\mathbf{c}$ gives

$$
\mathbf{c}=\mathbf{d} /\left(\mathrm{M}^{\prime}-1\right) \text {. }
$$

The distance of the image from the lens is then obtained from $z^{\prime}=\mathbf{b}-\mathbf{c}$, where $\mathbf{b}$ is the distance of the mesh from the lens.

In general the image exhibits some spherical aberration, which manifests itself as a change in shadow magnification as a function of the height $\rho$ of the ray at the lens, or the angle $\alpha^{\prime}$ at which the ray goes through the image. A plot of $\left(M^{\prime}-1\right)^{-1}$ versus $\rho^{2}$ leads to the paraxial value of $M^{\prime}$, and to the paraxial image 


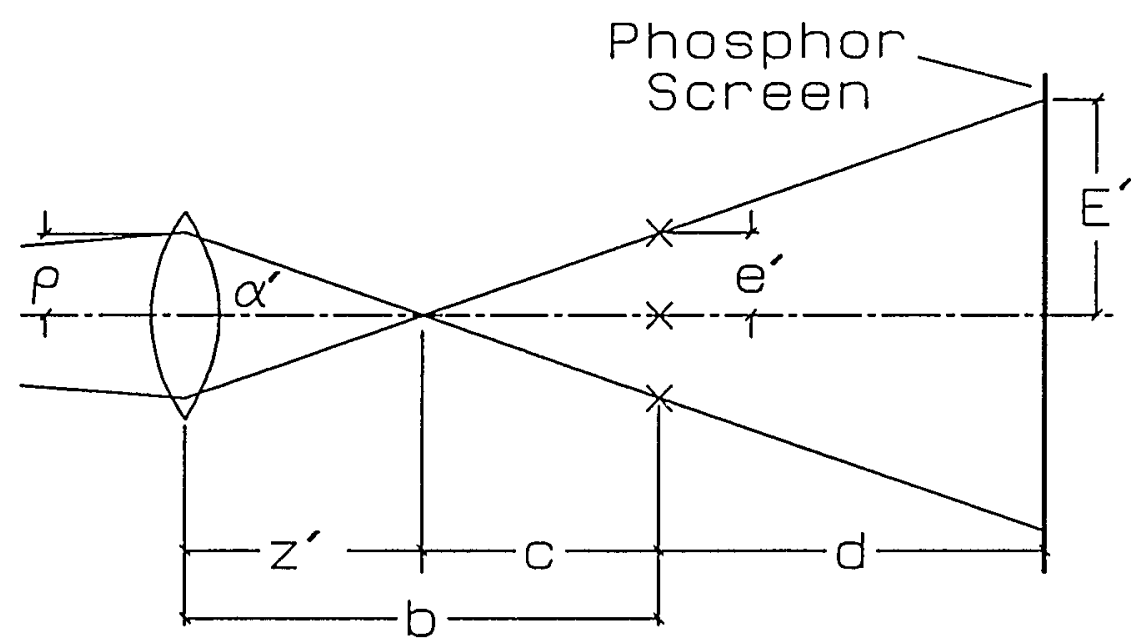

Figure 7. Diagram illustrating the relation between the magnification of the shadow cast by the mesh and the location of the image formed by the objective lens.

distance. The slope of this plot can also be used to determine the longitudinal spherical aberration $\Delta_{\mathrm{s}} \mathrm{z}^{\prime}$ in the image.

To test for chromatic aberration a small voltage increment $\Delta \mathrm{V}_{\mathrm{a}}$ is added to the accelerating voltage $V_{a}$ (independently of the lens and mirror voltages), and the change in magnification of the shadow pattern is used to determine the longitudinal chromatic aberration $\Delta_{\mathrm{c}} \mathrm{z}^{\prime}$ of the paraxial image. From equation (41) the longitudinal aberration is

$$
\Delta_{\mathrm{c}} \mathrm{z}^{\prime}=-\Delta \mathrm{c}=\mathrm{c}\left[\Delta\left(\mathrm{M}^{\prime}-1\right)\right] /\left(\mathrm{M}^{\prime}-1\right)=\mathrm{c} \Delta \mathrm{M}^{\prime} /\left(\mathrm{M}^{\prime}-1\right) .
$$

(For a mesh upstream from the image the signs of $\mathrm{M}^{\prime}$ and $\mathrm{c}$, and the type of shadow distortion, are reversed.) The resultant chromatic aberration coefficient for the system is

$$
\mathrm{C}_{\mathrm{c}}=\Delta_{\mathrm{c}} \mathrm{z}^{\prime} /\left(\Delta \mathrm{V}_{\mathrm{a}} / \mathrm{V}_{\mathrm{a}}\right) \text {. }
$$


In the case of undercorrected aberration, $z$ ' increases as the accelerating voltage increases, and $\mathrm{C}_{\mathrm{c}}$ is positive. If the mesh is downstream from the image, as in Figure 7, the shadow magnification increases. The opposite is true for overcorrected chromatic aberration.

The definition of the chromatic aberration coefficient given by equation (43) is based on the longitudinal chromatic aberration in the image. An alternative definition for the chromatic aberration coefficient is based on the radius of least confusion referred to object space, $r_{\ell c}=C_{c}(\Delta V / V) \alpha[33]$. In balancing the aberrations of the optical components in a system it is important to be consistent, because the alternative definition gives a value which is one-half of the value given by equation (43). We find equation (43) to be more meaningful and useful than the alternative definition because the longitudinal aberrations carry plus or minus signs and are the quantities which must cancel for aberration correction.

The correction of the final image of Figure 6 depends on the cancellation of the lens aberrations by the mirror aberrations. The aberrations of the relay lenses and the condenser lens can be ignored if the magnification of the image on which they operate is sufficiently high relative to the magnification of the image in the mirror stage. In this case the mirror need compensate only the aberrations due to two imagings by the interface lens and one imaging by the objective lens, and the condition for correction of chromatic aberration is

$$
\mathrm{C}_{\mathrm{cM}}+2 \mathrm{C}_{\mathrm{c} 2}+\left(\mathrm{m}_{1} \mathrm{~m}_{2}\right)^{2} \mathrm{C}_{\mathrm{c} 1}=0 .
$$


The subscripts 1 and 2 denote the objective and interface lenses, respectively, and $\mathrm{M}$ denotes the mirror. The lens aberration coefficients are referred to the low magnification sides of the lenses: the probe side of the objective lens, and the mirror side of the interface lens. The magnifications $m_{1}$ and $m_{2}$ are defined for a beam directed from the objective stage to the mirror. For this direction of the beam $m_{1}$ is greater than 1 , and $m_{2}$ is less than 1 . The product $m_{1} m_{2}$ is the magnification of the image in the mirror stage relative to the probe, and is usually not very different from unity in our experiments.

\section{APPARATUS}

The experimental study was carried out in a horizontal electron optical bench. The cover of the bench is a removable glass plate, which enables access to, and viewing of, the experiment. The bench was modified to include a double V-way section to support the optical components for the separated branches of the incident and reflected beams. The electron source (a thermionic triode gun) and condenser lens were supported on one track, and the objective lens and the fine-mesh assembly on the other track. A horizontal Y-shaped block was provided to hold the relay lenses. The interface lens and the mirror were supported on a single V-way. The magnetic deflectors were located at the intersections of the relay axes with the axes of the mirror and the condenser and objective branches. All of the lenses were electrostatic. The accelerating voltage was $15 \mathrm{kV}$. The lens voltages were obtained by means of a voltage divider from 
the high voltage power supply used for acceleration. The mirror bias was obtained from an auxiliary isolated power supply connected to the negative terminal of the accelerating voltage supply. The bias was adjustable from 0 to -5 $\mathrm{kV}$. The magnetic deflecting fields were produced by electromagnetic coils. The required voltages and currents were supplied through feedthroughs at the back and at the left end of the bench. A manipulator on the front of the bench was used to move the fine-mesh assembly along the objective axis for optimum adjustment and to ensure that the placement of the mesh, upstream or downstream from the image, was correctly identified. The shadow patterns cast by the mesh were received on a phosphor-coated fiber-optics output window at the right end of the bench, and recorded by a video camera. The operating pressure in the bench was about $5 \times 10^{-5}$ torr $\left(6.5 \times 10^{-3} \mathrm{pa}\right)$. A photograph of the optical bench is shown in Figure 8.

An engineering drawing of the hyperbolic mirror used in the present experiments is shown in Figure 9. The negative, or mirror, electrode has the shape of the conical asymptotic equipotential surface of the hyperbolic field. The mirror electrode is biased negatively with respect to the cathode of the electron gun, and electrons are reflected at curved equipotential surfaces in front of the electrode. The small opening at the vertex of the mirror electrode is for beam alignment. The anode has the curvature of a hyperbolic equipotential surface except for the beam-opening on the axis. The effect of the anode opening is to create a thin diverging lens. The length $\ell_{M}$ from the vertex of the mirror 


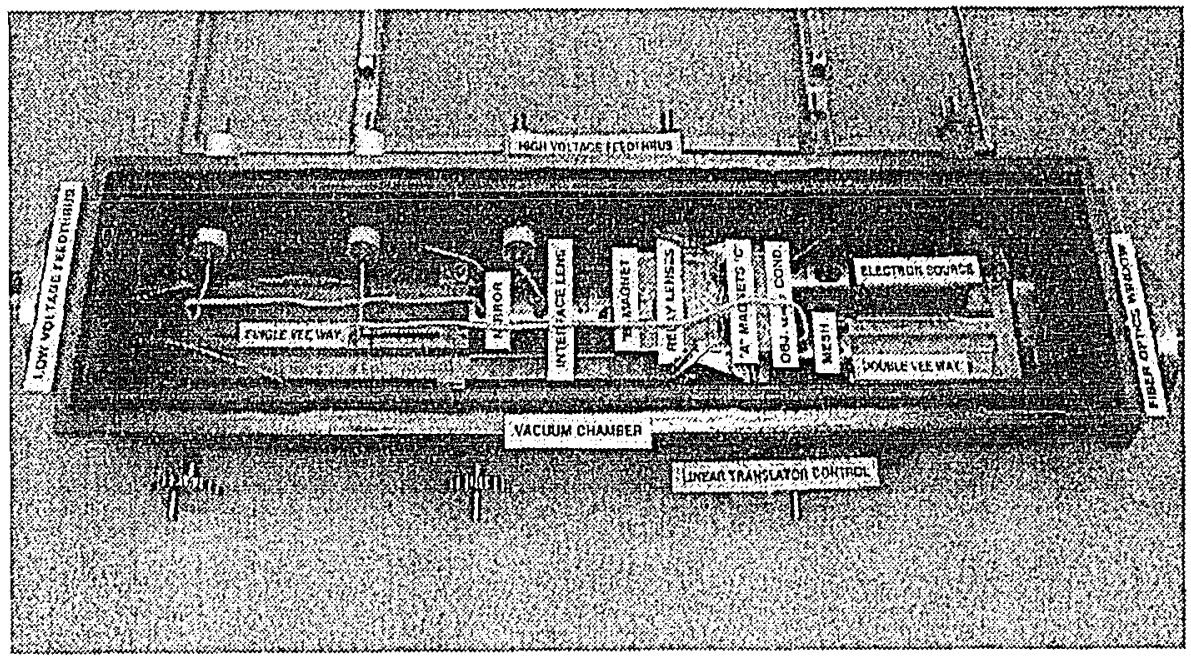

Figure 8. Photograph of the aberration-correction experiment set-up in the electron optical bench. The double V-way is at the right. The electron source and condenser lens are on the back track. The objective lens and mesh holder are on the front track. The Y-block, which houses the relay lenses, is in the middle. The single V-way, which supports the interface lens and the mirror, is on the left. The first and third magnetic deflectors are under the right edge of the Yblock, on their respective tracks. The second deflector (the separating magnet) is under the left edge of the Y-block, on the single V-way. The shadow patterns are received on a phosphorcoated fiber-optics window at the right end of the bench. One of the manipulators on the front of the bench is used to move the mesh holder along the track. Feedthroughs on the back bring in the high voltages, and feedthroughs at the left end on the bench are used for the low voltage supplies.

electrode to the inner surface of the anode is $2.54 \mathrm{~cm}$.

For the objective lens we used an electrostatic objective lens designed for a photoelectron microscope (PEM) or a low energy electron microscope (LEEM), and also suitable for a probe (Figure 10). In these experiments the center electrode of the lens was at the potential of the electron gun cathode, and the outer electrodes were grounded. The focal length of the lens at this voltage ratio 


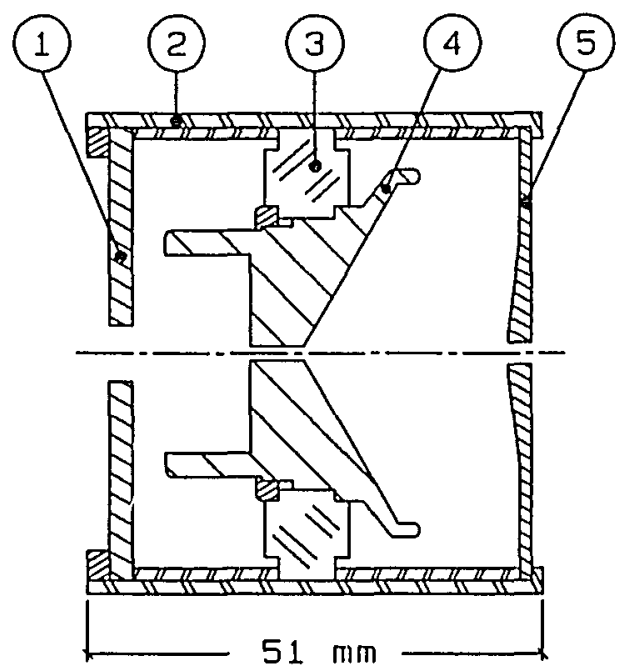

Figure 9. A scale drawing of the hyperbolic mirror used in the experiments. The anode (1) is in contact with the housing (4), and is grounded. The mirror electrode (2) is supported and isolated at a negative potential by the insulator (3). The grounded plate (5) has no function other than to terminate the potential field. The mirror electrode is biased negatively relative to the electron source so electrons do not reach it, but are turned back at a curved potential surface in front of the electrode. The conical surface of the mirror electrode has a slope of $\sqrt{2}$, which, along with the hyperbolic curvature of the anode, establishes a hyperbolic mirror field. The diverging effect of the aperture in the anode is taken into account separately. The aperture at the vertex of the mirror electrode is for alignment purposes.

is $10 \mathrm{~mm}$, and the working distance is $5 \mathrm{~mm}$. The low-magnification-side chromatic aberration coefficient is $3.4 \mathrm{~cm}$ at the magnification $\mathrm{m}_{1}=7.2$ used in the experiment.

A conventional projection lens [32] was used as the interface lens. Adjustments in the voltage ratios of the interface lens and the mirror were coordinated so that the image formed by the lens remained centered in the 


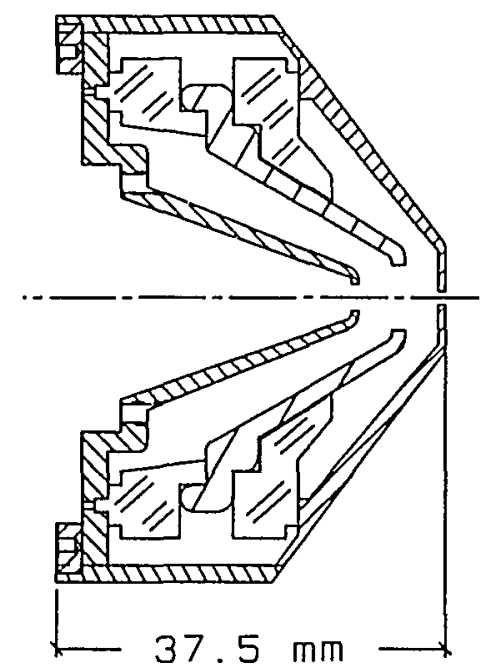

Figure 10. A scale drawing of the objective lens used in the aberration-correction experiments. The lens was designed for use at $30 \mathrm{kV}$ in a photoelectron microscope.

separating field. For given positions of the mirror and interface lens, the chromatic aberration in the final image was determined for selected values of the voltage ratios.

\section{RESULTS AND DISCUSSION}

Three pairs of shadow patterns are shown in Figure 11. They were recorded for the same positions of the lenses and the mirror, but with different values of the voltage ratios for the interface lens and the mirror. The objective lens was placed with its optical center $8.14 \mathrm{~cm}$ from the center of the deflecting magnet on the objective axis. The optical center of the interface lens was $16 \mathrm{~cm}$ from the center of the separating magnet on the mirror axis. The distance between the interface lens and the vertex of the mirror was $5.6 \mathrm{~cm}$. In Figure 11a 
$16.6 \mathrm{~mm}$

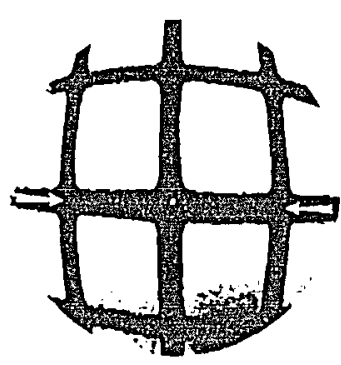

$15.9 \mathrm{~mm}$

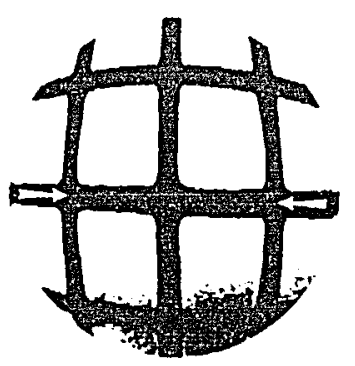

a
$17.6 \mathrm{~mm}$

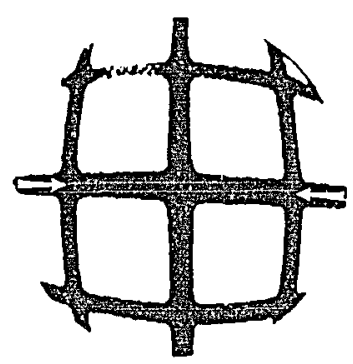

$17.6 \mathrm{~mm}$

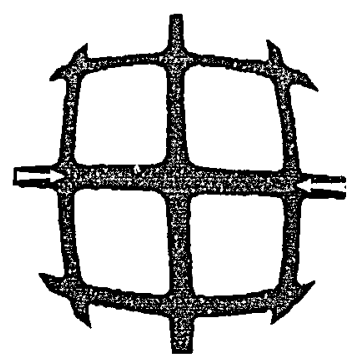

b
$16.7 \mathrm{~mm}$

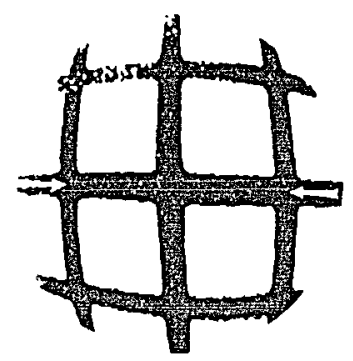

$17.2 \mathrm{~mm}$

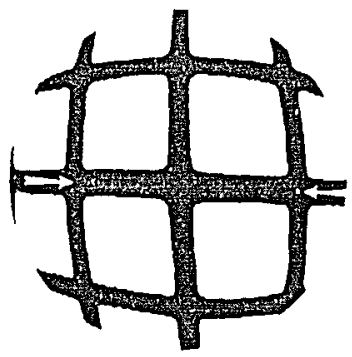

C

Figure 11. Shadow patterns indicating several stages of chromatic aberration correction: (a) undercorrection, (b) correction, (c) overcorrection. The shadow patterns were cast by a 1000/inch $(250 / \mathrm{cm})$ mesh closely following the demagnified image shown in Figures 6 and 7. In the chromatic aberration tests the beam voltage used for the upper patterns was larger by a small increment than for the lower patterns, a difference of $18 \mathrm{~V}$ for (a) and $9 \mathrm{~V}$ for (b) and (c). The mean accelerating voltage was $15 \mathrm{kV}$. The lens and mirror voltages did not change. A slightly larger magnification of the upper pattern in (a) indicates undercorrection, whereas the slightly smaller magnification in (c) indicates overcorrection. The magnifications are approximately equal in (b) indicating correction of chromatic aberration.

the upper pattern corresponds to an additional 9 volts of accelerating voltage (the lens and mirror voltages remained unchanged) and the lower pattern corresponds to a reduction of 9 volts, a difference of 18 volts. In Figures $11 \mathrm{~b}$ and $11 \mathrm{c}$ the difference in accelerating voltage for the upper and lower shadow patterns is 9 
volts rather than 18 volts. A 1000 squares/inch $(394 / \mathrm{cm})$ mesh was located downstream from the image, and at a distance of $37 \mathrm{~cm}$ in front of the phosphor screen. The diameter of the shadow patterns shown corresponds to $2.7 \mathrm{~cm}$ on the phosphor screen. The average paraxial shadow magnification at the phosphor screen was 333. From equation (41), the distance from the image to the mesh was $0.111 \mathrm{~cm}$. The pair of shadow patterns on the left (Figure 11a) shows a slightly larger magnification in the upper pattern than in the lower pattern, indicating that the image moved forward (i.e., away from the lens) as the beam voltage was increased and that the chromatic aberration was undercorrected. The magnification difference is about $4.3 \%$. From equation (42), $\Delta_{\mathrm{c}^{\prime}} \mathrm{z}^{\prime}=48 \mu \mathrm{m}$, and from equation (43), $\mathrm{C}_{\mathrm{c}}=(48 \mu \mathrm{m}) /\left(18 / 15 \times 10^{3}\right)=4.0 \mathrm{~cm}$. The patterns in the center (Figure 11b) have approximately equal magnifications, indicating that chromatic aberration is approximately corrected. On the right (Figure 11c), the magnification of the upper pattern is $3 \%$ smaller than that of the lower pattern, indicating that the image moved backward (toward the lens) as the voltage was increased and that the chromatic aberration was overcorrected. In this case $\Delta_{c} z^{\prime}$ $=-33 \mu \mathrm{m}$, and $\mathrm{C}_{\mathrm{c}}=(-33 \mu \mathrm{m}) /\left(9 / 15 \times 10^{3}\right)=-5.6 \mathrm{~cm}$. The voltage ratios and magnifications for the mirror and the interface and objective lenses corresponding to the three sets of patterns in Figure 11 are given in Table I.

The barrel-type distortion in the shadow patterns of Figure 11 indicates that the image has undercorrected spherical aberration. However, a substantial amount of spherical aberration correction accompanies the chromatic aberration 
correction. For comparison, considerably more distortion can be seen in the shadow pattern of Figure 12, which was obtained with the objective lens alone (without the correcting system) under otherwise similar conditions. The lower distortion in Figure 11 makes it feasible to position the mesh closer to the image, and to use smaller voltage increments for exploring chromatic aberration. Some asymmetry in the shadow patterns occurs because there was no provision for centering a mesh opening or intersection on the optical axis.

Cancellation of the chromatic error, as evidenced by the shadow patterns of Figure 11b, amounts to correcting an electron probe having the configuration used in the experiment. This same method of investigating the conditions for chromatic aberration correction can be applied to a transmission electron microscope (TEM) with the electrons traveling backward through the system and focusing to a probe in the specimen plane, as in Figure 6, but with an objective lens suitable for a TEM. If the specimen plane is immersed in a magnetic field, as is usual in electromagnetic TEMs, the effect of the magnetic field on the shadow patterns will need to be taken into account. However if the specimen plane is in field-free space the shadow pattern analysis of Section 3. can be applied directly. In the case of emission electron microscopes and related lowenergy electron microscopes, the specimen is the cathode of an accelerating field, and correction of the final image of Figure 6 would still leave the aberrations of the accelerating field uncorrected. For correction of this type of microscope, the 
final image of Figure 6 should be overcorrected by the amount needed to compensate the aberration of the accelerating field.

The foregoing experiments were carried out under conditions which

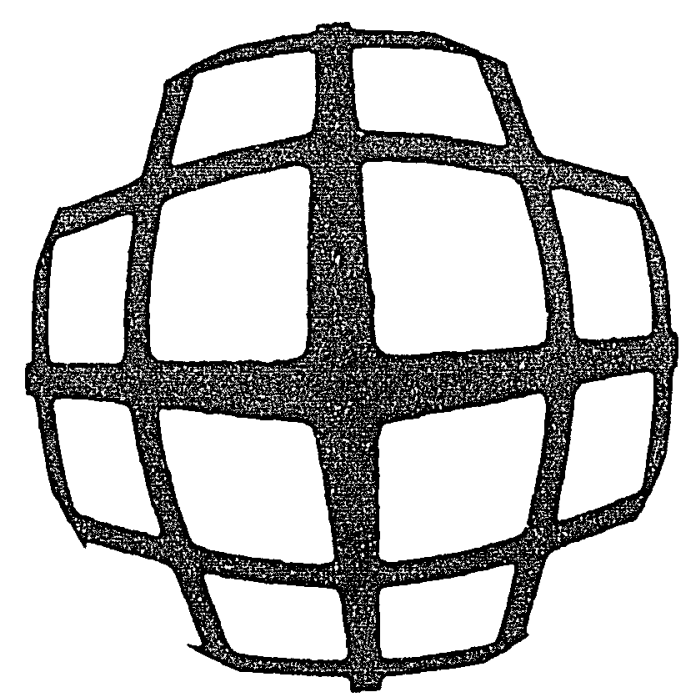

Figure 12. Shadow pattern obtained under conditions similar to those for Figure 11, but without the correcting system.

differed somewhat from the conditions assumed theoretically. One difference was that, because of space limitations in the optical bench, the magnification of the intermediate image in the relay branches was not high enough for the relay aberrations to be neglected, as assumed for equation (44). Another difference was that, although the beam alignment was fairly good, it was not accurate enough for the effect of misalignment on the aberration coefficients to be ignored. Misalignment decreases the magnitude of the mirror aberrations and, to a lesser extent, increases the lens aberrations. The electron gun was not equipped with the lateral controls needed for precise alignment. For the given lens 


\section{TABLE I}

\section{MIRROR AND LENSES}

\begin{tabular}{|c|c|c|c|c|c|}
\hline \multicolumn{2}{|c|}{ Objective Lens } & \multicolumn{2}{|c|}{ Interface Lens } & \multicolumn{2}{|c|}{ Mirror } \\
\hline $\begin{array}{l}v_{1} \\
1.00\end{array}$ & $\begin{array}{l}\mathrm{m}_{1} \\
7.2\end{array}$ & $\begin{array}{l}v_{2} \\
1.67\end{array}$ & $\begin{array}{l}\mathrm{m}_{2} \\
1 / 4.9\end{array}$ & $\begin{array}{l}v_{M} \\
0.963\end{array}$ & $\begin{array}{l}\mathrm{m}_{\mathrm{M}} \\
1.0\end{array}$ \\
\hline 1.00 & 7.2 & 1.89 & $1 / 3.16$ & 0.993 & 1.0 \\
\hline 1.00 & 7.2 & 1.94 & $1 / 2.85$ & 0.996 & 1.0 \\
\hline
\end{tabular}

Note. The voltage ratios and magnifications of the mirror and the interface and objective lenses corresponding to the shadow patterns in Figure 11. The quantity $v$ is the ratio of the accelerating voltage, $V_{a}=V_{A}-V_{C}$, to the voltage applied to the lenses and the mirror, $\mathrm{V}_{\mathrm{A}}-\mathrm{V}_{\mathrm{i}=1,2, \mathrm{M}}$. $\mathrm{V}_{\mathrm{A}}$ is the potential of the anode (in our experiments $\left.V_{A}=0\right)$ and $V_{C}$ is the potential of the cathode of the electron gun; $V_{1,2, \text { or } M}$ is the potential of the negative electrode of the objective lens, interface lens, or mirror respectively. The magnifications $m_{1}$ and $m_{2}$ are defined for a beam directed from the objective stage to the mirror, as in a TEM, and are the reciprocals of the magnifications $m_{1 p}$ in the probe mode. The ratio for the mirror was larger (mirror bias smaller) than predicted from equation 44 because the aberrations of the relay lenses were not negligible, and the beam alignment was not accurate enough to avoid the effect of misalignment on the aberration coefficients of the mirror.

magnifications and voltage ratios in Table I, the experimental values obtained for the mirror voltage ratio are larger (mirror bias smaller) than predicted from the values of $C_{c M}$ in ref. [28] for an aligned beam. Nevertheless Figure 11b shows that the chromatic aberration of a lens system can be compensated by means of a mirror, and that the separating system works well, with very little astigmatism. These experiments have provided incentive and justification for continuing our studies with an improved version of the experimental apparatus capable of providing simultaneous correction of chromatic and spherical aberration, as proposed in [28], and tests of the expected resolution. 


\section{CHAPTER IV}

\section{SIGNIFICANCE OF RESULTS}

Using the mirror to correct chromatic aberration in an electron optical system but without fully compensating spherical aberration: what results can be anticipated? The two most common figures of merit for optical systems are resolution and intensity. Correction of aberrations will improve resolution and increase the intensity available at the output of electron optical systems. The magnitude of the benefit will depend upon the relationship between the size of the aberrations and the resolution in the system in question. In this chapter, three examples of electron optical systems will be examined in some detail for the expected benefits of the correction of chromatic aberration.

One measure of resolution in light instruments is the Rayleigh criterion which states that two adjacent equally luminous image points are just resolved if the intensity in their overlapping patterns drops by about $25 \%$ of the peak intensity of either point. This criterion is also used to evaluate resolution in electron optics. Another method of evaluating resolution is the Taylor's criterion where the resolution between two overlapping luminous points is defined to be the distance between the peaks which causes the sum of the overlapping intensities to be equal to half of the intensity at the peak. 
According to the geometric theory of resolution developed by Rempfer and myself [33], the image of a small uniformly illuminated disc object is a peaked cone in the plane of best focus. The half-maximum diameter of the intensity pattern referred to object space is nearly equal to the diameter of the object. The intensity of the peak also increases with the size of the object. However, the intensity of the maximum is reduced by the effect of aberrations. Resolution and intensity are seen to be related in this theory in that, in finite-time microscopy the illumination in the image peak must be sufficient that a non-noisy image can be recorded in the allotted exposure time. Under the Rayleigh criterion, the resolution turns out to be roughly equal to the diameter of the smallest object supplying the required illumination in the image peak. The question of limiting resolution in electron optics frequently becomes one of image contrast in the presence of background illumination. For an image to be seen, it must rise above the background illumination. A contrast requirement which is often used is that in order for two luminous points to be resolved, their overlapping intensities added to the background must drop by $10 \%$ of the maximum intensity of one peak added to the background. The technique developed in reference [33] to calculate the intensity in the image will be used to evaluate the geometrical resolution results for the following cases. 


\section{TRANSMISSION ELECTRON MICROSCOPE}

One of the oldest and most familiar electron optical instruments is the transmission electron microscope (TEM). In this case the spacial resolution in the image is of primary importance. Intensity in the final image is usually adequate, being supplied by an electron source and not the specimen itself. In the example given for resolution limited by diffraction in Chapter I, a $60 \mathrm{kV}$ beam of electrons has a wavelength of $.05 \AA$ and, with an angular aperture of 0.01 rad., yields a resolution of about $3 \AA$ in an electron microscope. Since the aberrationlimited resolution is much larger than the ultimate diffraction limit of $0.61 \lambda / \sin \alpha$ $=0.03 \AA$, the benefits of reducing the magnitude of aberrations would be expected to benefit the resolution.

In order to apply any criterion to the image of a luminous object in the TEM, it is necessary to know how aberrations affect the distribution of intensity. This relationship is supplied by the geometrical theory of resolution. In calculating TEM resolution directly more information about the specifıc imaging conditions of contrast and detection limits would have to be known. In order to estimate the improvement expected from eliminating chromatic aberration for a given imaging condition however, the situation is less difficult. In the plane of best focus the current in the image of a uniformly illuminated element of radius $=\mathrm{R}$, formed by a lens system of spherical aberration $\mathrm{C}_{\mathrm{s}}$ with a single energy beam, is

$$
I \propto 1.2 R^{8 / 3}\left(4 / C_{s}\right)^{2 / 3}
$$


Whatever the particular conditions under consideration are, the intensity will be smeared out along the axis by the chromatic error, lowering the value of current contained in the smallest resolved image. Knowing the reduction in current, the resulting effect on resolution can be estimated by solving this relationship for the new value of $\mathrm{R}$ containing the same intensity formerly producing sufficient contrast to constitute the resolution limit. If instead, the resolution element $\mathrm{R}$ is resolved in the presence of chromatic aberration and we ask what smaller value of $\mathrm{R}$ would contain the same current in the absence of chromatic aberration, we may estimate this improvement from the same equation if we know the amount by which the current is increased.

Spherical error also smears the image of each point in the object out along the axis in the image. The technique described in reference [33] allows the intensity distribution in the image of a geometrical resolution element to be calculated in the presence of spherical error. The effect of chromatic aberration is to further smear each monochromatic image plane along the axis by an amount $d z$ given by $d z=C_{c} d V / V$ where $C_{c}$ is the chromatic aberration, $d V$ is the change in beam voltage, and $\mathrm{V}$ is the beam voltage. For a thermionic source of electrons $\mathrm{dV}$ might be 0.5 volt. With a beam voltage $\mathrm{V}=40 \mathrm{kV}$, and $\mathrm{C}_{\mathrm{c}}=1.2 \mathrm{~cm}, \mathrm{dz}=$ $1500 \AA$. In this case the spherical aberration constant is equal to $4 \mathrm{~cm}$ and a $5 \AA$ radius resolution element would have maximum intensity imaged in the plane of best focus. This plane would be displaced from the paraxial image plane by about $4070 \AA$. The reduction in peak intensity for a further displacement of the image 
along the axis caused by chromatic error would result in a decrease of peak intensity in the image to about $58 \%$ of that in the plane of best focus. Conversely, eliminating chromatic error from a system suffering from chromatic error should result in an increase in intensity of $1 / .58=1.71$ times the peak intensity in the plane of best focus. The intensities are smeared along the axis by chromatic error reducing the peak intensity but not appreciably widening the distribution. We may then take the increase in peak intensity to calculate a new resolution $\mathrm{R}$ from the relationship for the total current in the element just resolved by the system. An increase in intensity of 1.71 corresponds to an improvement of resolution from $10 \AA$ to about $8.2 \AA$. Thus, only a modest improvement for an electron microscope results from correcting chromatic aberration due to the energy distribution in the electron source. However, if the energy distribution due to scattering by the specimen is considered, a much larger benefit could be expected.

\section{EMISSION MICROSCOPE}

In the case of emission microscopy the specimen emits electrons or ions under the influence of illumination by photons or bombardment by electrons or ions. The specimen is placed in an electric field and the emitted electrons are accelerated and imaged at magnification with an optical system much the same as in the case of the TEM. The presence of the accelerating field introduces both undercorrected spherical and chromatic aberrations. The magnitude of the 
chromatic aberration for the accelerating field is of the same order as the objective lens aberration but the chromatic error of the field can be much larger than that due to the lens, particularly in the case of small emission energies. These aberrations add to those of the objective and other lenses in the system further limiting the final resolution. In the photoelectron microscope (PEM) [2], the present practical limit of the microscope is about $100 \AA$. Theoretical considerations set the diffraction limit to about $20 \AA$. Depending upon the emission energy of the photoelectrons, the benefits of correcting chromatic aberration alone may range from improving the resolution to $30 \AA$ at low emission energies and good contrast to $80-90 \AA$ with higher emission energies and poor contrast. For the PEM where many biological structures lie in a range of sizes near the theoretical resolution limit, any improvement in resolution would be welcome. Implementing the mirror as described in this study in such a system would already provide some compensation of spherical error. Most likely any attempt to add the mirror to an emission microscope would seek to correct both aberrations simultaneously thereby realizing the full capability of the microscope.

\section{ELECTRON OR ION PROBE}

The electron and ion probes are instruments designed to produce a small focussed beam of electrons or ions. The optics of the probe are distinguished from the TEM in that it is usually necessary to have the probe focussed at some distance from the final lens in order to accommodate some other equipment. By 
requiring the final lens to have a large working distance, the resulting aberrations of the lens are greatly enlarged. An electrostatic probe lens having a working distance of $5 \mathrm{~cm}$ and operating at unity magnification might have spherical aberration $C_{s}=400 \mathrm{~cm}$ and chromatic aberration $C_{c}=40 \mathrm{~cm}$. If the source of current has a radius of $75 \AA$ then in the plane of best focus the image of about 75 $\AA$ in half width will contain a current limited by the spherical error. The axial displacement of the image produced by a voltage spread in the source of 0.5 volt in a beam voltage of $10 \mathrm{kV}$ will be $2 \times 10^{5} \AA$. A shift in the image of this magnitude would reduce the peak current to $37 \%$ of the value in the plane of best focus. Furthermore, with such a large chromatic aberration in this plane the image would be spread out to the place where the half width was four times the original size with a very slowly diminishing tail spreading current well beyond the desired image. The benefit of eliminating the chromatic aberration is too large to estimate using this method. By superimposing a number of image planes corresponding to monochromatic images of the source, the spread in chromatic differences could be made small enough forl each plane to form an integrated total image of the source and the result of eliminating chromatic aberration estimated. Given the rather slowly varying character of the image peak with axial location due to the large spherical error, and the peaked nature of the Maxwellian distribution of current from a thermionic source, I would expect a nearly linear increase in current in the image with respect to reduction of chromatic error. Attempting to estimate the resolution by comparing the areas under graphs of the 
intensity distributions, I would expect an order of magnitude increase in current into the image in the plane of best focus by eliminating the chromatic aberration. The trend toward lower energy probes in several areas of biological and semiconductor research suggests that this would be a project worthy to pursue. Whatever the benefits are in the example examined here, the benefits will be larger as the beam voltage is further reduced.

\section{CONCLUSION}

In these three examples: the TEM could benefit from correction of chromatic aberration due to the electron energy spread caused by inelastic scattering by the specimen; the emission microscope would also benefit significantly because of the large chromatic aberration coefficient of the accelerating field; and the probe would receive enormous benefit due to the large chromatic coefficients of the lenses needed to obtain the required working distance. The magnitude of the chromatic error relative to the limiting resolution was the determining factor in evaluating the potential improvement available. Since the chromatic error scales up proportional to the focal length of electron lenses, one could anticipate that everywhere that long focal lengths are required in electron optics, resolution would be limited to aberrations and there would be potential benefit for using a mirror to correct the chromatic aberration. Besides the probe, electron optical systems with long focal lengths are found in ion implanters, mass spectrometers, surface ion mass spectrometers (SIMS), electron 
energy loss spectrometers (EELS) of a variety of forms, electron beam furnaces and welders, image converters, vidicons, cathode ray tubes (CRT), and television display tubes. It seems reasonable that these instruments would be potentially eligible for adaptation to mirror correction of chromatic aberration in the form presented in this work. The greatest motivation for the correction of aberration in any of the systems mentioned would be where increased sensitivity or resolution lies at the threshold of important new information as in the case of the PEM. The correction of chromatic aberration alone would seem to be most beneficial in those cases where the chromatic error was the major resolution limiting factor. All probes meet this test. Improvement in the resolution of probes to the limit of spherical error can be achieved with the mirror as used in this study. Ion probes, where the ions are formed with a large energy spread, are even better candidates for the correction of chromatic aberration. Many of the analytical instruments also meet this criterion. There is constant need to improve instrument sensitivity in order to solve increasingly more difficult problems lying at the threshold of detection. Detecting the presence or effects of contaminants in the environment continues to challenge instrumentation to detect at ever smaller limits. Every increment in improving the sensitivity of these instruments permits the exploration of previously undetectable conditions. The mirror, implemented as it was used in this study, can provide immediate benefit to a many of these needs. 


\section{REFERENCES}

[1] L. W. Swanson, G. A. Schwind, and A. E. Bell, J. Appl. Phys. 51 (1980) 3453.

[2] G. F. Rempfer and O. H. Griffith, Ultramicroscopy 27 (1989) 273.

[3] O. H. Griffith and W. Engel, Ultramicroscopy 36 (1991) 1.

[4] W. H. Fincham, Optics (Hatton Press, London, 1956) pp. 240-241.

[5] M. Herzberger, Modern Geometrical Optics (Interscience Publishers, London, 1958) pp. 112-113.

[6] Von W. Henneberg and A. Recknagel, Zeitschr f. Physik 12 (1935) 621.

[7] A. Recknagel, Z. Phys. 104 (1936) 381.

[8] Von G. Hottenroth, Ann. der Physik 30, no. 5 (1937) 689.

[9] W. Henneberg, U. S. Patent No. 2,161,466 (6 June 1939).

[10] V. K. Zworykin, G. A. Morton, E. G. Ramberg, J. Hillier, and W. A. Vance, Electron Optics and the Electron Microscope (Wiley, New York, 1945). See especially pp. 456-464, 630-631, and 645.

[11] O. Scherzer, Zeit. f. Phys. 101 (1936) 593.

[12] O. Scherzer, Optik 2 (1947) 114.

[13] H. Koops, Proc. 9th Intern. Congr. on El. Microscopy, ed. J. M. Sturgess (Microsc. Soc. of Canada, Toronto, 1978), III 185.

[14] A. V. Crewe and D. Kopf, Optik 55 (1980) 1-10.

[15] L. R. Harriott, W. L. Brown, and D. L. Barr, Am. Vac. Soc. 8, no. 6, (1990) 1706.

[16] G. M. Fleming, U. S. Patent No. 2,740,919 (3 April 1956). 
[17] M. Kuzuya, T. Hanai, M. Hibino, and S. Maruse, J. El. Micr. 31, no. 1 (1982) 18-26.

[18] P. W. Hawkes, Adv. in Electronic and Electron Phys. Suppl., ed. A. Septier (Academic, New York, 1980), 13A, 109-140.

[19] W. Hawkes and E. Kasper, Principles of Electron Optics (Academic Press, 1989), 857-878.

[20] E. G. Ramberg, J. Appl. Phys. 20 (1949) 183.

[21] R. Castaing and L. Henry, C.R. Acad. Sci., Paris 255 (1962) 76.

[22] M. S. Mauck, Master's Thesis, Portland State University (1969).

[23] J. M. Lafferty, Proc I.R.E. 35 no. 8 (1947) 778.

[24] R. M. Henkelman and F. P Ottensmeyer, J. Phys. E 7 (1974) 176.

[25] H. Lichte and G. Mollenstedt, J Phys E: Sci. Instrum. (1979) 941.

[26] J. P. van der Merwe, J. Appl. Phys. 52, no. 7 (1981) 4411.

[27] G. F. Rempfer and M. S. Mauck, Proc. Ann. EMSA Meet. 43 (1985) 132.

[28] G. F. Rempfer, J. Appl. Phys. 67 (1990) 6027.

[29] O. H. Griffith, K.K. Hedberg, D. Desloge, and G. F Rempfer, J. of Micr. 168, part 3 (1992) (in press).

[30] C. J. Davisson and C. J. Calbick, Phys. Rev. 42, 580 (1932).

[31] G. F. Rempfer and M. S. Mauck, Optik (1992)(in press).

[32] G. F. Rempfer, J. Appl. Phys. 57 (1985) 2285.

[33] G. F. Rempfer and M. S. Mauck, J. Appl. Phys. (1988) 2187.

[34] G. D. Archard and T. Mulvey, J. Sci. Inst. 35 (1958) 279-283.

[35] W. Telieps and E. Bauer, Ultramicroscopy 17 (1985) 57-66. 
[36] A. B. Bok, J. B. Le Poole, J. Roos, and H. deLang, Adv. Opt. El. Micr., ed. R. Barer and V. E. Cosslett (Academic, New York, 1971) 4161.

[37] E. Kasper, Optik $28(1968 / 9) 54$.

[38] G. F. Rempfer and M. S. Mauck, Proc. Ann. EMSA Meet. 44 (1986) 886.

[39] G. F. Rempfer, Ultramicroscopy (in press). 
APPENDIX A

THE BEAM-SEPARATING SYSTEM 
The aberration-correcting properties of electron mirrors have been known at least as far back as 1935 [6]. One of the reasons that the application of a mirror to an electron microscope has been hindered has been the problem of separating the incident and reflected beams from the mirror without introducing resolution-limiting errors. A number of beam-separating methods are considered in references [18] and [19]. Most of these separating methods are based on the use of magnetic fields. Beams of electrons traveling in opposite directions can be separated by means of a transverse magnetic deflecting field, as illustrated in Figure 13a. However, the magnetic deflecting field introduces its own aberrations which include chromatic dispersion and "deflection defocussing", and a compression of the beam at right angles to the plane of deflection.

Pole-pieces can be designed to reduce deflection aberrations [34]. Such is the case in the mirror microscope described by Telieps and Bauer [35] where the beams incident on the mirror and returning from the mirror are separated by a single magnetic deflecting field. A stigmator can be used to correct for compression aberration. Bok et al. [36] used a stigmator in conjunction with their separating system where magnetic deflectors are used in a bridge arrangement. Another method employing a mirror with a superimposed axial magnetic field in conjunction with the objective lens was proposed by Kasper [37] for correction of a transmission electron microscope.

The separating system developed for this work is also based on magnetic deflection. The detrimental effects of deflection aberrations on resolution that others 

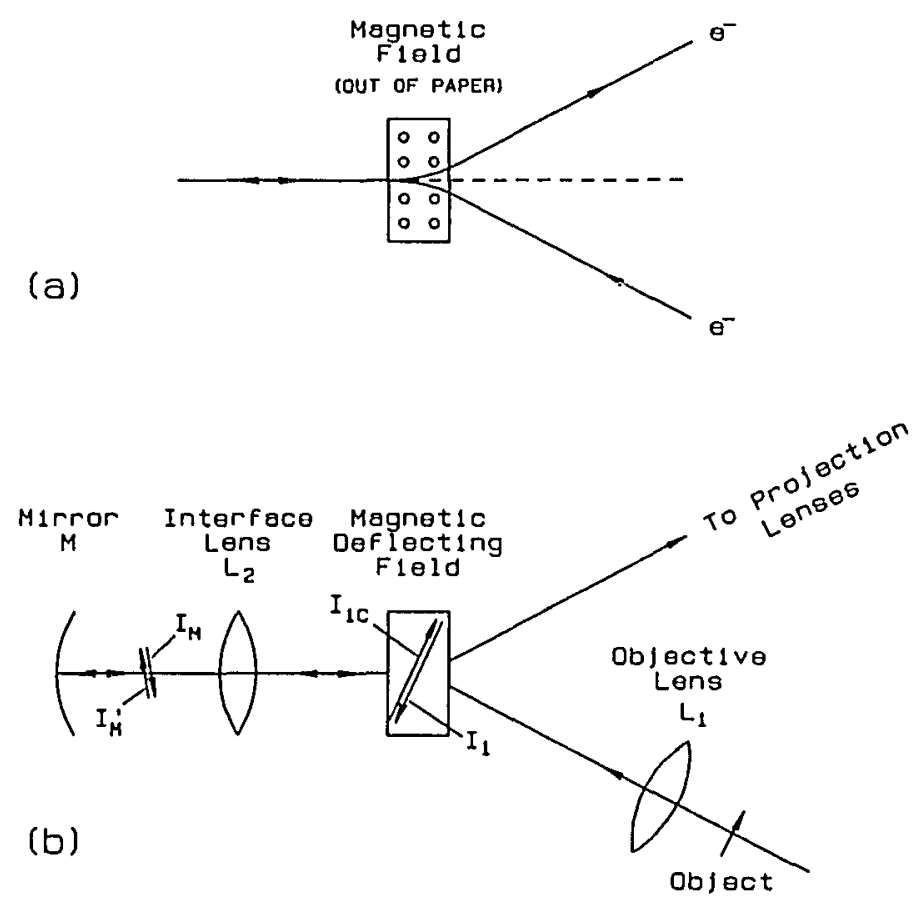

Figure 13. Separation of incident and returning beams by a magnetic field. (a) A transverse magnetic field separates electrons traveling along the same axis in opposite directions on the left into two separate diverging axes on the right. (b) A magnetic deflecting field for separating the incident and returning beams at the plane of the images $I_{1}$ and $I_{1 C}$.

have experienced are avoided by arranging to have deflections take place at image planes [38]. Subsequent images formed in the system are then brought into focus by intervening lenses regardless of any dispersion or compression of the beam caused by the deflector. Higher order aberrations introduced from deflections can be further reduced by deflection in magnified image planes. These considerations point to the plane of the magnified images $I_{1}$ and $I_{1 c}$ of Figure 13 as the ideal location for a magnetic deflector to separate the incident and returning beams. 
With the mirror operating in the symmetric mode, the object and image planes coincide. The lens $L_{2}$ then relays the mirror's object/image into the separating magnet. The image in the magnet remains fixed at the center of the deflecting field while the voltage parameters on the mirror and lens $\mathrm{L}_{2}$ are adjusted together for aberration correction of the entire lens system.

A circular cross-section for the magnetic deflecting fields is advantageous. Since the image being transferred through the deflector occupies only a small area near the center of the field, the beams enter and leave the deflection field substantially at right angles to the boundary, thus avoiding the compression effects of the fringing fields. In addition each ray heading for the center of the magnet traverses an equal distance in the magnetic field and exits appearing to come from the same point at the center of the field. For electrons of the same energy, equal paths in the field produce equal angular deflections and results in the absence of keystone distortion in the image common in other deflection geometries. Since all of the rays exiting from the magnet appear to come from the center, there is no deflection of the image position with variations of magnetic field strength. This feature makes the image position insensitive to magnet current and accelerating voltage power supply variations, an important consideration in high resolution applications. Additionally, since the rays entering the magnet cross in the center, small linear misalignment of the magnet location is compensated by the fact that rays shortened the most entering are shortened the least exiting, tending to make the ray paths and deflections equal. 
This auspicious geometry appears to be particularly suitable for use with imaging systems employing electron mirrors.

An additional concern involves the orientation of the image in the separating field. The images $I_{1}$ and $I_{1 c}$ shown in Figure $13 \mathrm{~b}$ formed by the objective lens are not at right angles to the axes of the mirror or the projection lenses. If the axes of the objective lens, mirror, and projection lenses can be arranged to be parallel to each other this short-coming can be avoided. To do so requires two deflections of the beam between the objective axis and the mirror axis, and two deflections between the mirror axis and the projection axis, as illustrated in Figure 6. In order for each of these deflection to take place at an image plane, lenses are required in each leg, between the deflection fields. In order to produce images at right angles to the mirror and projection axes, a pair of relay lenses producing a real intermediate image is used to transfer the images between deflectors. The magnification of the images on which the relay lenses operate can be made large enough that the aberrations in the relay stages are negligible.

Whereas a magnetic field is required for separation of the electron beams, either magnetic or electric fields can be used for the other two deflectors. The insensitivity of the deflector developed here to misalignment and power supply variations has led to the incorporation of this design in all three deflection locations of the optical system used in this study. By making the objective, mirror, and projection axes parallel as shown in Figure 5, all three axes could be mounted 
vertically. Vertical orientation is to be preferred in order to minimize the sensitivity of a microscope to vibrations. The permissible levels of vibrations and other spurious disturbances become more stringent as the resolution limit is improved and is a serious concern in seeking to take advantage of the aberration correction potential of the mirror. While correction of aberrations is a necessary requirement for achieving higher resolution, it is not the only requirement, and the correction of the primary aberrations will leave higher order effects to set the ultimate limit to resolution.

All that can be said for the benefits of using the deflection geometry described here for electron beams can also be said for ion beams. With the sign of the potentials changed on the source, the lenses, the deflectors and the mirror, the correction system performs the same function with ions as for electrons. The only element in this system that depends upon the ratio of charge to mass is the magnetic deflection. Here, because the deflection is small, the magnetic field can be increased sufficiently to allow passage of ions. 
APPENDIX B

\section{DETAILS OF THE EXPERIMENT}


This appendix contains additional details describing the equipment used in the work reported in Chapter III and the description of the experimental procedure employed in the study.

\section{APPARATUS}

\section{The Optical Bench}

The experimental study was carried out in the horizontal electron optical bench shown in the photograph in Figure 8. The optical bench consists of an airtight cast brass enclosure mounted on a vacuum pumping station. The top of the bench is sealed with a removable glass plate. The glass allows viewing of the experiment in progress and aids in the initial alignment of the experiment. The interior surface of the optical bench was lined with sheets of Mu-metal for shielding from stray magnetic fields. The bench ordinarily contains 3 sections of a 1-meter straight V-way but was modified, replacing one of the single sections at one end with a double V-way section. The double V-way was used to support the optical components for the separated branches of the incident and reflected beams for this study. The V-ways are machined to support and align the 2.125 inch diameter electron optical components used in our laboratory. The electron source (a thermionic triode gun) and condenser lens were supported on one track, and the objective lens and the fine-mesh assembly on the other track. A horizontal Y-shaped block was constructed to hold the relay lenses. The interface lens and the mirror were supported on the remaining single $\mathrm{V}$-way. The magnetic 
deflectors were located at the intersections of the relay axes with the axes of the mirror and the condenser and objective branches. Sensitive areas of the beam path were covered with additional mu-metal covers to further reduce the effects of stray magnetic fields on the beam alignment. The only openings in the magnetic shielding were those to provide electrical connections to components and viewing access to the phosphor alignment screens. The required voltages and currents were supplied to the interior of the bench through vacuum feedthroughs at the back and at the left end of the bench. These connections were sealed with rubber o-rings.

The only solid electrically insulating material used inside of the bench, including the high voltage mirror and lens insulators, was Rexolite. Rexolite is a cross-linked poly-styrene plastic having the jointly desirable features that it possesses the highest electrical resistivity of all plastics, is mechanically rigid, and outgasses only water vapor and carbon dioxide in vacuum.

\section{The Power Supply}

The accelerating voltage was supplied by a voltage regulated Computer Power Supply Model CPS-100-N 0 to $-30 \mathrm{kV}$ variable source fixed at $15 \mathrm{kV}$ throughout these experiments. A schematic of the electrical supply wiring is shown in Figure 14. The cathode, the source of electrons in the bench, was connected directly to the high voltage source (actually the center tap of the hairpin filament supply). A switch in the cathode circuit was used to insert one or two 9 volt batteries in series with the cathode for chromatic aberration 
measurements. The lens voltages were obtained by means of a voltage divider from the high voltage power supply. The mirror bias was obtained from a Bertan Model PMT-5A/N power supply. The anode of the mirror supply was connected directly to the negative terminal of the accelerating voltage supply. The mirror bias voltage was adjustable from 0 to $-5 \mathrm{kV}$ more negative than the electron source. Primary power was supplied to the mirror bias and source supplies via a

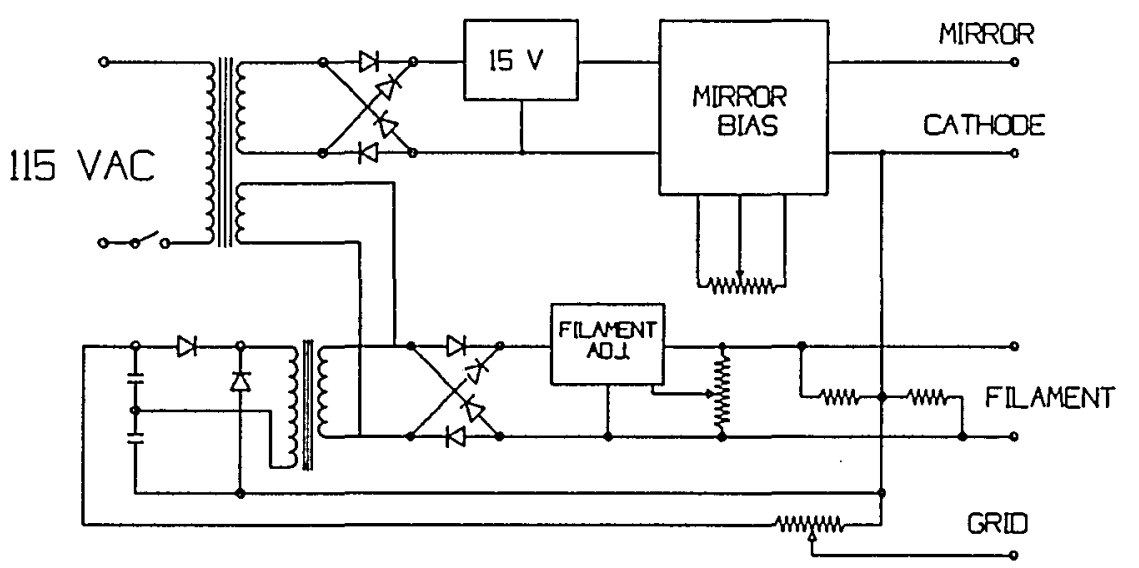

Figure 14. Schematic of power supplies for experimental study. Secondary of line transformer is wound with high voltage insulating wire for high voltage isolation.

line transformer constructed with an especially large window to accommodate low voltage secondary windings wound with high voltage insulated wire. High voltage isolation of these supplies was achieved in this way without resorting to expensive isolated supplies. The filament heating current was taken from its isolated winding on the transformer and passed through a rectifier and current control. The grid source bias control supply also received its power from the isolated 
transformer winding. The voltage required $(300 \mathrm{~V})$ was produced by a small filament transformer wired with its low voltage winding as the primary and its high voltage winding as the secondary. Direct current was produced with a voltage doubler rectifier and passed to a potentiometer control on its way to the grid.

High voltages were measured with a Keithley Model 197 Autoranging Microvolt DMM using the Model 1600A $40 \mathrm{kV}$ high voltage probe. Since the cathode voltage was supplied from a voltage regulated source, the operating voltage was measured when set and verified only periodically. A scale on the 10turn mirror control knob was calibrated by measuring the mirror voltage with the voltmeter and measurements taken from the scale thereafter.

\section{Magnetic Deflecting Fields}

All three of the magnetic deflecting fields used in these experiments were produced by electromagnets. Each deflector consisted of a pair of coils wound on Rexolite plastic forms and supported by deflection yokes constructed to confine the deflecting field to the region of the electron beam and limit the stray magnetic fields. Mu-metal pole faces were adhesively attached to the pole pieces to insure uniform magnetic fields in the gap. The pole faces were electrically isolated from the pole pieces and wired to voltage sources to provide vertical electrostatic deflection for beam alignment. The pair of coils for each deflector was connected in series and each of the three deflectors was energized by an adjustable constant current supply. 


\section{$\underline{\text { Lenses and Mirror }}$}

All of the lenses used in this study were electrostatic. The interface lens, used in the mirror branch, transfers the image in the separating magnet to the mirror and back again. The aberrations of the interface lens are thus doubled since this lens acts on the beam travelling in both directions to and from the mirror. An electron microscope projection lens was used in this location. The projection lens properties were measured experimentally and are given in Table II for the range of lens voltage ratios used.

\section{EXPERIMENTAL PROCEDURE}

\section{The Vacuum System}

In order to obtain the measurements required to demonstrate the correction of chromatic aberration of the lens system with the hyperbolic electron mirror, the following procedure was used. At the beginning of each experimental session the optical bench was evacuated. The process required approximately one hour beginning with roughing out the bench and vacuum system with the mechanical vacuum pump. The diffusion pump was first evacuated to about $10^{-2}$ torr where it was valved off and the diffusion pump heater was turned on. When the pressure in the bench reached approximately $40 \times 10^{-3}$ torr, the bench and diffusion pump were pumped in parallel. After about 20 minutes heating, the diffusion pump was ready for use. The bench roughing line was valved off and the high vacuum valve between the diffusion pump was slowly opened while the pressure was monitored 
TABLE II

PROJECTION LENS PROPERTIES

\begin{tabular}{ccccc}
\hline$\nu$ & $\mathrm{f}_{0}$ & $\mathrm{~g}_{0}$ & $\mathrm{C}_{\mathrm{f}}$ & $\mathrm{C}_{\mathrm{g}}$ \\
2.00 & 1.854 & 1.659 & 2.75 & 2.28 \\
1.82 & 1.401 & 1.285 & 3.11 & 2.56 \\
1.67 & 1.061 & 1.007 & 3.22 & 2.76 \\
1.54 & 0.831 & 0.797 & 2.74 & 2.75 \\
& & & & \\
\hline
\end{tabular}

Note. Here the paraxial focal length $\mathrm{f}_{0}$ and focal distance $\mathrm{g}_{0}$ as well as the chromatic aberration coefficients are given. The quantity $\mathrm{C}_{\mathrm{c}}$ is obtained from $\mathrm{C}_{\mathrm{f}}$ and $\mathrm{C}_{\mathrm{g}}$ and magnification using the relationship $\mathrm{C}_{\mathrm{c}}=\left[\left(1+\mathrm{m}^{2}\right) \mathrm{C}_{\mathrm{g}}+2 \mathrm{mC}_{\mathrm{rl}} \mathrm{O}\right.$. The voltage ratio is given by $v=\left(V_{A}-V_{C}\right) /\left(V_{A}-V_{L}\right)$.

in the diffusion pump roughing line. Pressure in the roughing line was kept below $70 \times 10^{-3}$ torr in order to keep the diffusion pump from overloading and backing oil vapor into the bench. When the high vacuum line was fully opened, the bench required some 20-30 minutes more to reach operating pressure.

\section{The Optical Design}

In order to establish the approximate conditions of aberration correction, the theoretical properties of the mirror were combined with the previously empirically determined properties of the lenses. Using the Gaussian thick lens formula $(g-z)\left(g^{\prime}-z^{\prime}\right)=f^{2}$ where $z$ and $z^{\prime}$ are the object and image distances and $g$ is the focal point distance and starting with the electron source, we determined the voltage ratio (the ratio of the accelerating voltage to the voltage on the lens) for each lens. Since both the focal position and focal length were known for discrete values of voltage ratio, a graphical construction was utilized for the solution where 
quantities $\mathrm{f}^{2}$ and $(\mathrm{g}-\mathrm{z})\left(\mathrm{g}^{\prime}-\mathrm{z}^{\prime}\right)$ were plotted against voltage ratio. The intersection of these lines determined the expected operating point of the lens. The experimentally determined properties of each lens were used for these calculations.

The condenser and interface lens properties were taken from a previous study [32]. The properties of the relay lenses were determined in a similar way at the beginning of this study. Similarly, the objective lens properties were measured in our optical bench for the voltage ratio $=1$ used in this study.

For the calculation of the mirror location, the theoretical image location values were used. By combining the distances between optical elements and focal properties, voltage ratios for each lens and the mirror were determined. The voltage divider, which consisted of twenty 20 -megohm resistors connected in series, was prepared for the experiments by replacing individual resistors with potentiometers of equal resistance at those places where calculations indicated taps would be necessary to operate particular lenses. The lenses and mirror were connected to the appropriate taps with high-voltage insulated wire. The wires were fitted with banana plugs attached to acrylic insulator rods so that voltages could be changed while the high-voltage was on. This was a valuable asset in locating a wayward electron beam and was useful in alignment verification.

\section{The Alignment Procedure}

With the physical locations and voltage ratios determined for the lenses and mirror and the optical bench evacuated, the high voltage was turned on and the 
filament current was brought up until the filament was yellow-white in color. The grid voltage was advanced until light from the first phosphor alignment screen in front of the condenser lens could be seen. Necessary adjustments to the filament location controls were made to center the beam on the condenser screen.

Current to the first turning magnet was applied and adjusted until the beam could be seen on the second screen on the entrance to the first relay lens. The magnet current was adjusted to center the beam in this opening by enlarging the beam with the condenser lens (by raising its voltage ratio along the voltage divider momentarily) and centering the illuminated beam around the relay lens opening. Current was turned up in the second magnet until the beam could be found on a screen placed just beyond this magnet. In some cases where the magnets had been replaced or otherwise rewired, the current had to be reversed through the magnet in order to obtain the correct deflection direction. (In some preparatory experiments the second magnet was replaced with a phosphor screen in order to verify the proper focussing of the relay lens pair.) The same procedure was performed with the second magnet to center the beam in the following aperture. At this point the beam was falling on the interface lens. As the current in the second magnet was adjusted to sweep the beam across the interface lens opening, the beam could be seen to sweep across the screen in front of the mirror. The beam was centered on the opening to the mirror. The mirror voltage was brought up slowly and a beam could be seen on the following screen placed at the rear of the interface lens (mirror side). (Here or earlier the filament grid bias was 
adjusted for the peak illumination on one of these intermediate phosphor screens and the filament current adjusted for adequate brightness.) The beam was thus traced back to the second magnet. Adjustment of the mirror or interface voltage revealed the centering of the beam on the entrance to the second relay lens pair. At this point the symmetry of the three intersecting axes determined the beam alignment. Some exercise was required to mechanically align these three axes. Mechanical repositioning of the relay lens assembly with metal shims was tried until satisfactory alignment was obtained. Since the electrostatic deflectors in the magnets were in image planes for the beam, little or no displacement of the beam was found for these controls and the alignment of the second relay lens pair had to be done mechanically. A number of electrostatic and magnetic alignment fixtures were designed, constructed and tested in order to alleviate this problem. The final results were obtained with a combination of mechanical alignment and a small lateral voltage applied to the second magnet where the pole faces had been divided in two along the mirror axis.

During most of the investigations where sufficient room was allotted between the third magnet and the objective lens, a small phosphor screen could be swung into the beam with one of the mechanical manipulators. Investigation of the beam at this location disclosed the characteristic caustic of the beam according to the magnitude and sign of the spherical aberration present. This was particularly useful in verifying the predicted behavior of the mirror and the functional relationship of the correction formulas developed here. (As an aside, the 
behavior of the caustic on this screen stimulated reproducing this behavior with light optics and resulted in two additional papers $[33,39]$ and a new geometrical theory of resolution.) Initially it was hoped that spherical aberration could be corrected simultaneously with chromatic aberration. Observations of the beam incident on the objective lens vindicated predictions of this possibility but coarseness in the beam alignment capabilities of the experimental apparatus precluded achieving our best results of simultaneous correction. As the beam could be observed prior and following the objective lens, the beam could be seen to go from overcorrected to undercorrected behavior under the influence of the objective lens alone, further reinforcing our predictions.

\section{EXPERIMENTAL RESULTS}

Once the beam was satisfactorily aligned in the bench and the lenses were set to the expected voltages, the mirror voltage was brought up until illumination could be seen on the final screen. The location of a 1000/inch mesh was varied along the axis until a high magnification shadow of the mesh could be seen on the output screen. By translating the mesh along the axis of the beam, its location with respect to the caustic could easily be found. The magnification would gradually grow as the mesh moved toward the caustic, the mesh bars disappearing altogether as the whole beam passed through one opening in the mesh(!), and reappearing on the other side gradually growing smaller as the mesh retreated from the caustic. Distortion patterns associated with the magnitude and sign of 
the spherical aberration could also be seen as the mesh was translated along the beam. (Pincushion distortion for the mesh between the lens and caustic with ordinary undercorrected spherical aberration and barrel distortion for overcorrected spherical aberration. $\mid$ The distortion types are reversed for the mesh positioned beyond the caustic.)

Chromatic aberration was studied by observing change in the shadow magnification with incremental changes in the electron beam accelerating voltage. For example, with the mesh positioned beyond the final image, if an increase in beam voltage was accompanied by an increase in magnification, this indicated that the image had moved toward the mesh and away from the lens, and that the chromatic aberration was undercorrected. A decrease in magnification for the same change in voltage would indicate over-correction.

When the optical arrangement was such that illumination on the final screen was too dim to view satisfactorily, an image intensifier was used to increase illumination. The intensified shadow patterns could be imaged with a CCD video camera and viewed on a monitor. These patterns were recorded on a video cassette recorder. Results were photographed off of the monitor with a $35-\mathrm{mm}$ camera and measurements made on prints from these pictures. Calibration of the final image size was accomplished by ratioing the measurements of the mesh on the final photograph to the image intensifier diameter on the photograph. The diameter of the image intensifier output screen was 1.125 inches. 